\title{
PBX transcription factors drive pulmonary vascular adaptation to birth
}

\author{
David J. McCulley, ${ }^{1}$ Mark D. Wienhold, ${ }^{1}$ Elizabeth A. Hines, ${ }^{2}$ Timothy A. Hacker, ${ }^{3}$ Allison Rogers, ${ }^{3}$ Ryan J. Pewowaruk, ${ }^{4}$ \\ Rediet Zewdu, ${ }^{5}$ Naomi C. Chesler, ${ }^{1,4}$ Licia Selleri, ${ }^{5,6}$ and Xin Sun ${ }^{2,7}$ \\ DDepartment of Pediatrics, ${ }^{2}$ Laboratory of Cenetics, ${ }^{3}$ Department of Medicine, and ${ }^{4}$ Department of Biomedical Engineering, University of Wisconsin - Madison, Madison, Wisconsin, USA. ${ }^{5}$ Department of \\ Cell and Developmental Biology, Weill Medical College of Cornell University, New York, New York, USA. ${ }^{6}$ Program in Craniofacial Biology, Institute of Human Cenetics, Departments of Orofacial Sciences and \\ Anatomy, UCSF, San Francisco, California, USA. Department of Pediatrics, UCSD, San Diego, California, USA.
}

A critical event in the adaptation to extrauterine life is relaxation of the pulmonary vasculature at birth, allowing for a rapid increase in pulmonary blood flow that is essential for efficient gas exchange. Failure of this transition leads to pulmonary hypertension (PH), a major cause of newborn mortality associated with preterm birth, infection, hypoxia, and malformations including congenital diaphragmatic hernia (CDH). While individual vasoconstrictor and dilator genes have been identified, the coordination of their expression is not well understood. Here, we found that lung mesenchyme-specific deletion of CDHimplicated genes encoding pre-B cell leukemia transcription factors $(P b x)$ led to lethal PH in mice shortly after birth. Loss of $P b x$ genes resulted in the misexpression of both vasoconstrictors and vasodilators in multiple pathways that converge to increase phosphorylation of myosin in vascular smooth muscle (VSM) cells, causing persistent constriction. While targeting endothelin and angiotensin, which are upstream regulators that promote VSM contraction, was not effective, treatment with the Rho-kinase inhibitor Y-27632 reduced vessel constriction and PH in Pbx-mutant mice. These results demonstrate a lungintrinsic, herniation-independent cause of $\mathrm{PH}$ in $\mathrm{CDH}$. More broadly, our findings indicate that neonatal PH can result from perturbation of multiple pathways and suggest that targeting the downstream common effectors may be a more effective treatment for neonatal PH.

\section{Introduction}

Early fetal detection and improvements in medical and surgical management of complex developmental defects have resulted in increased survival among infants born with previously lethal anomalies. Congenital diaphragmatic hernia (CDH) is a common congenital anomaly that occurs in 1 in 3,500 to 5,000 live births $(1,2)$. Despite improvements in the care of infants with $\mathrm{CDH}$, the mortality rate associated with the disease continues to be $20 \%$ in isolated cases and $50 \%$ in complex cases (3-5). The primary cause of the high mortality rate is a lethal combination of pulmonary hypoplasia and pulmonary hypertension $(\mathrm{PH})(6,7)$. The severity of these defects is highly variable among patients. Furthermore, the $\mathrm{PH}$ that frequently leads to lethality in patients with $\mathrm{CDH}$ is often not responsive to available medications (8-11).

The longstanding model of $\mathrm{CDH}$ is one that originates from malformation of the diaphragm, and, secondarily, herniation of the abdominal organs into the chest causes compression of the lungs and pulmonary vasculature, preventing their normal development. However, the severity of lung hypoplasia and $\mathrm{PH}$ and the rate of mortality in patients with $\mathrm{CDH}$ exceed what is observed in infants with other malformations, such as congenital pulmonary airway malformation or omphalocele, which simi-

Conflict of interest: The authors have declared that no conflict of interest exists Submitted: February 14, 2017; Accepted: November 7, 2017 Reference information: J Clin Invest. 2018;128(2):655-667. https://doi.org/10.1172/JCI93395. larly compromise lung development due to mechanical compression $(12,13)$. These findings raise the possibility that there may be diaphragm-independent causes of lung abnormalities in $\mathrm{CDH}$. Indeed, lung growth and patterning defects have been observed in animal models of $\mathrm{CDH}$ prior to normal diaphragm closure (14-19). However, there has been no clear in vivo demonstration as yet that $\mathrm{PH}$ can arise independently of the diaphragmatic defect and herniation seen in $\mathrm{CDH}$.

Recent genomic sequence analysis of patients with $\mathrm{CDH}$, correlated with gene expression studies in the developing mouse diaphragm, has identified a growing list of candidate genes implicated in the pathogenesis of $\mathrm{CDH}(14,16,20-23)$. Among them are $P b x$ genes that encode homeodomain-containing transcription factors best known to act as Hox cofactors $(24,25)$. Global inactivation of $P b x 1$ in mice led to diaphragm malformation, supporting its involvement in $\mathrm{CDH}$ (26). In this study, we set out to address the role of $P b x$ in pulmonary vascular development, a process that is frequently impaired in patients with $\mathrm{CDH}$. Our findings support the model that mutation of $\mathrm{CDH}$ candidate genes can act within the developing lung to directly impair lung and pulmonary vascular development and function, independently of their role in diaphragm formation. Furthermore, our data illustrate the importance of identifying the causative genetic defect in patients with $\mathrm{CDH}$ and demonstrate how, in the mouse model, pharmacological manipulation of the altered downstream signaling cascades can be explored to reverse the lethal PH that affects infants with the disease. 
E12.5

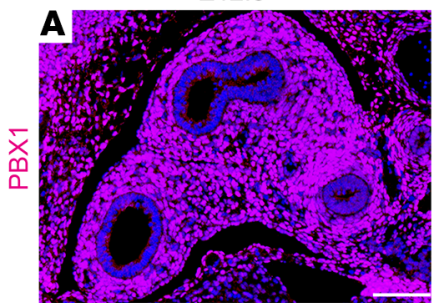

P3

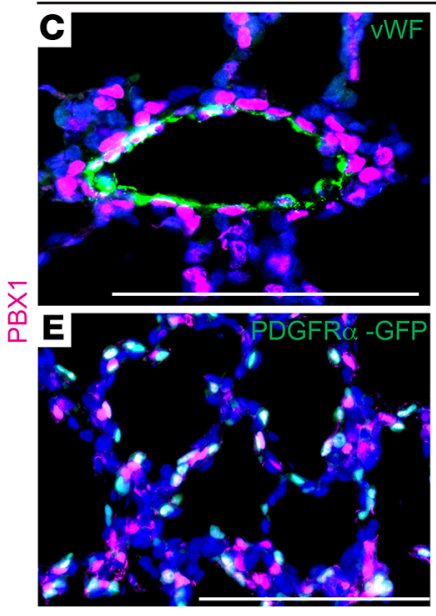

E12.5

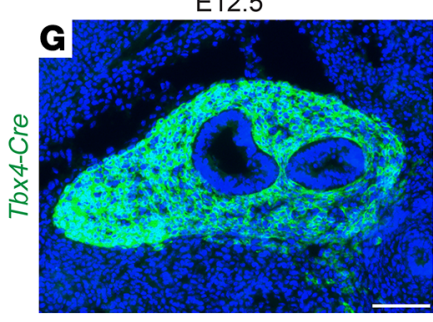

P3 Pbx1/2-CKO
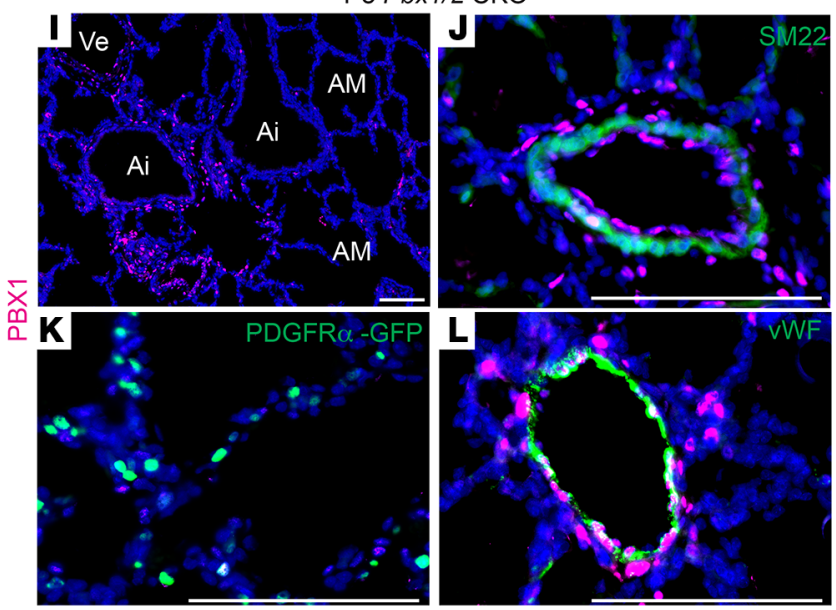

P3
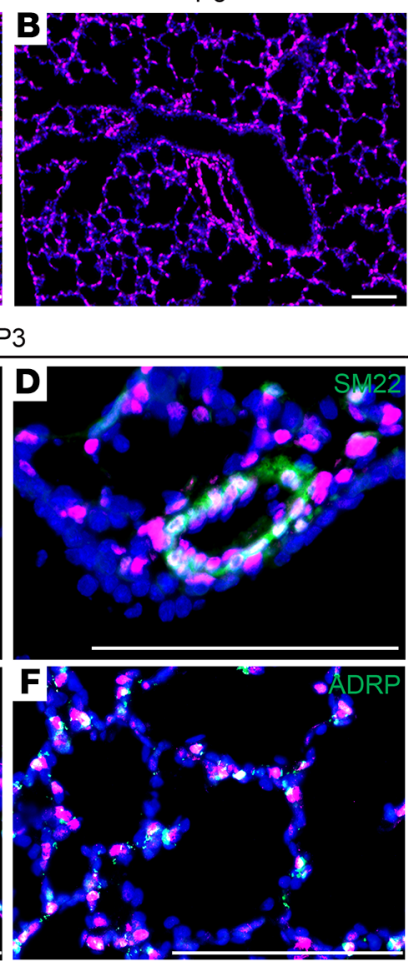

P3

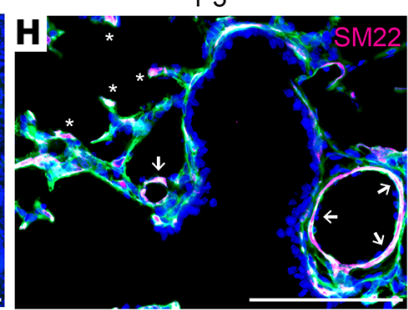

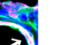

\section{(1)}

Figure 1. PBX1 is expressed in the lung mesenchyme. (A and B) PBX1 was broadly expressed throughout the lung mesenchyme at E12.5 through early postnatal stages. (C-F) PBX1 was expressed in multiple cell types in the lung mesenchyme including vWF-stained vascular endothelium, SM22-stained VSM, PDCFR $\alpha$-GFP-stained alveolar myofibroblasts, and ADRP-stained lipofibroblasts. ( $\mathbf{G}$ and $\mathbf{H}$ ) Tbx4-Cre had broad activity in the lung mesenchyme when crossed with a green fluorescent reporter and overlapped with PBX1 expression in SM22-stained alveolar myofibroblasts (indicated by asterisks) and VSM (indicated by arrows). (I) Using Tbx4 ${ }^{\text {cre }}$ to delete $P b x 1$ resulted in widespread loss of PBX1 in the mutant mouse as compared with the control lung mesenchyme in $\mathbf{B}$. Within the mutant, there was a more complete loss of PBX1 in the alveolar mesenchyme (AM) than in the peribronchial mesenchyme adjacent to central airways (Ai) and vessels (Ve). (J-L) Pbx1/2-CKO mice lacked PBX1 expression in SM22-stained VSM and PDGFR $\alpha$-GFP-labeled alveolar myofibroblasts but retained PBX1 expression in vWF-stained endothelium. Scale bars: $100 \mu \mathrm{m}$.

vascular smooth muscle (VSM) cells, alveolar myofibroblasts, and lipofibroblasts, as indicated by costaining with cell-specific markers (Figure 1, C-F). Both the level and pattern of PBX1 expression are maintained in the late embryonic and early postnatal lung. PBX1 is not expressed in epithelial type 1 or type 2 cells (Supplemental Figure 1, A and B; supplemental material available online with this article; https://doi.org/10.1172/JCI93395DS1).

Lung mesenchymal deletion of $\mathrm{Pbx} 1 / 2$ results in postnatal lethality, lung simplification, and cardiac hypertrophy. To determine whether $P b x$ genes play a cell-autonomous role in the lung, we applied a tissue-specific deletion approach, using a $T b x 4^{\text {Cre }}$ allele that has broad lung mesenchymal activity but no activity in the diaphragm (28). We found that $T b x 4^{C r e}$ induced widespread recombination in the lung mesenchyme by E12.5 (Figure 1G). This overlapped with the pattern of PBX1 expression in multiple cell types including VSM cells and alveolar myofibroblasts (Figure 1H, and Supplemental Figure 1, C-E). Notably, $T b x 4^{\text {Cre }}$ was not active within the vascular endothelial cells where PBX1 was also expressed (Supplemental Figure 1F).

As it has been reported that the PBX2 expression pattern is similar to that of PBX1 in a number of tissues including the lung mesenchyme (29), we inactivated $P b x 2$ in addition to $P b x 1$, generating $\mathrm{Tbx} 4^{\mathrm{Cre}} \mathrm{Pbx} 1^{\mathrm{fl} / \mathrm{Pl}} \mathrm{Pbx2^{-/ }}$ mutant mice (hereafter referred to as $\mathrm{Pbx1/2-CKO} \mathrm{mice;} \mathrm{CKO,} \mathrm{conditional} \mathrm{knockout)} \mathrm{(Supplemental}$ Figure 1G). Pbx1/2-CKO mice showed more complete deletion of PBX1 expression in the alveolar mesenchyme than in the peribronchial region (Figure 1I). As predicted from Cre activity, the mutant lungs retained PBX1 expression in vWF-expressing vascular endothelium but not in SM22-expressing VSM cells or alveolar myofibroblasts (Figure 1, J-L). Mice with homozygous inactivation of the $P b x 1$ conditional allele and heterozygously null for $P b x 2$ (Tbx $4^{\text {Cre }}$ $P b x 1^{\Re / / l} \mathrm{Pbx}^{+/-}$) were viable and appeared grossly normal (data not shown). Likewise, mice with heterozygous inactivation of the $P b x 1$ conditional allele and homozygously null for $\mathrm{Pbx} 2\left(\mathrm{~Tb} x 4^{\mathrm{Cre}} \mathrm{Pbx} \mathrm{1}^{\mathrm{Tl} /+}\right.$ $\left.P b x 2^{--}\right)$were grossly normal and used as controls.

As $T b \times 4^{\text {Cre }}$ is not active in the diaphragm (28), the diaphragm of $P b x 1 / 2-C K O$ mice developed normally (Supplemental Figure $1 \mathrm{H})$, unlike the diaphragmatic defects observed in $P b x 1$ global mutants (26). A previous study showed that conditional inactivation of $P b x 1$ in the lung, heart, brain and other tissues using Islet$1^{\text {cre }}$ led to lethality at birth with failed lung inflation, which was attributed to decreased surfactant expression (30). In contrast, Pbx1/2-CKO mice survived birth at the expected Mendelian ratio 

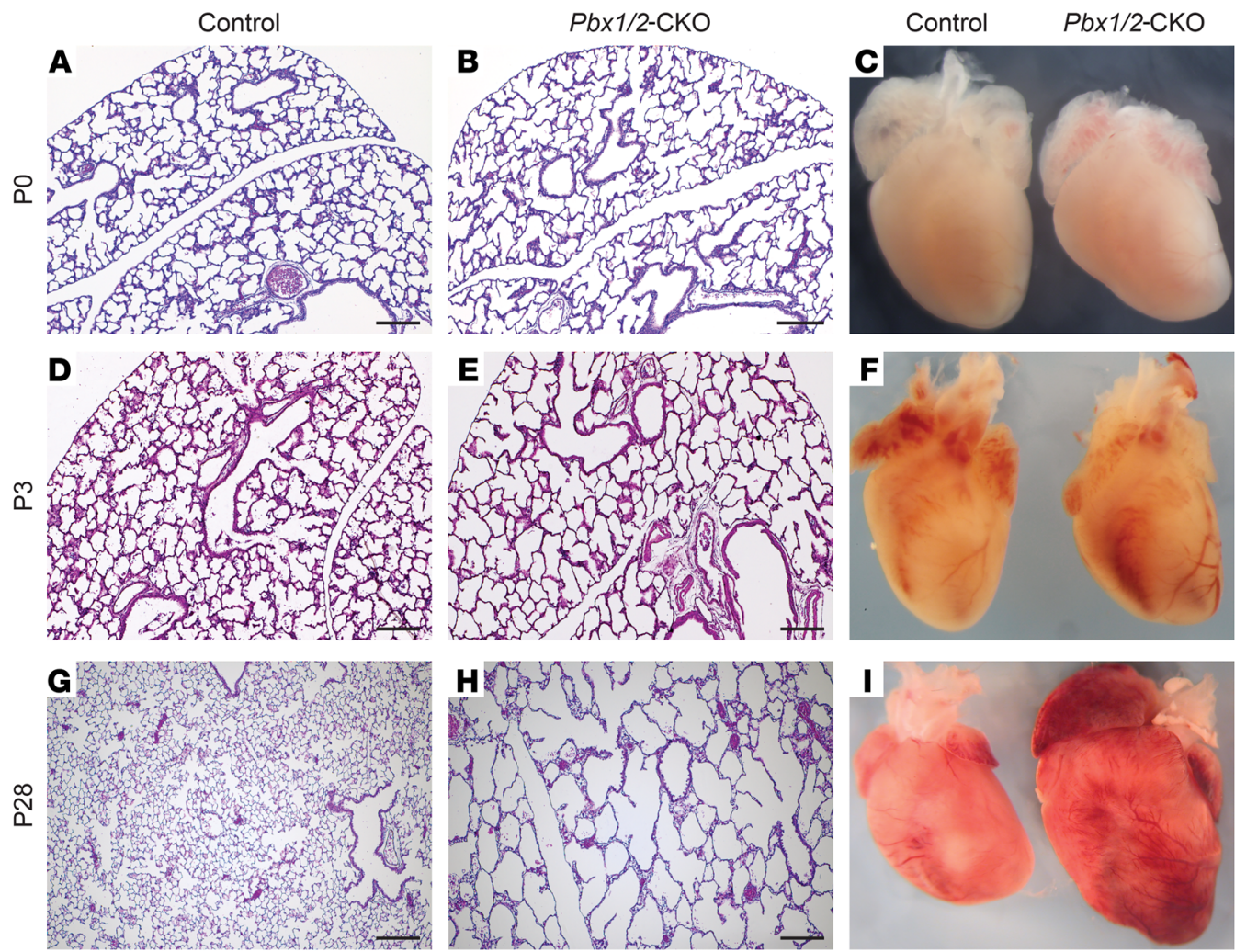

J

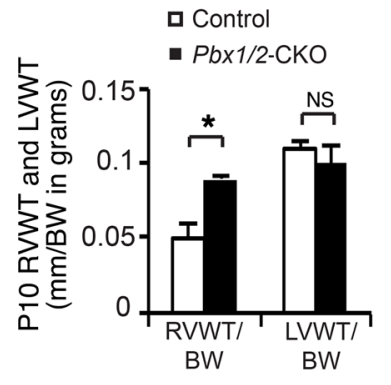

K

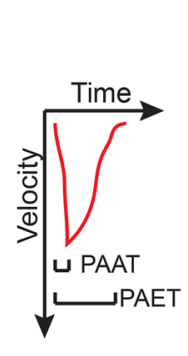

P7 PAAT

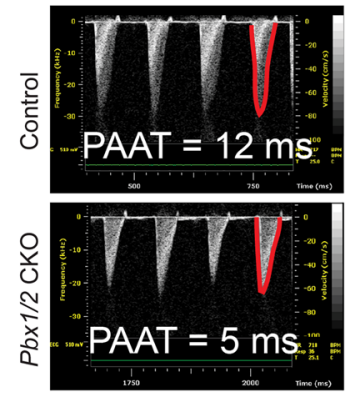

$\mathbf{L}$

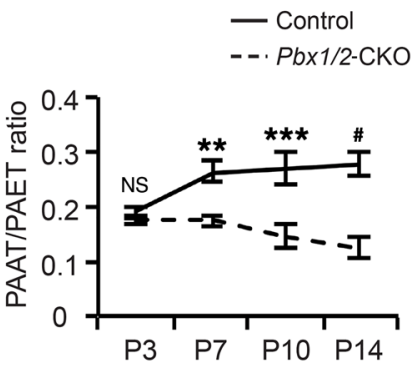

Figure 2. Pbx1/2-CKO mice exhibit abnormal postnatal lung development, progressive cardiac hypertrophy, and PH. (A-C) At birth, $P b \times 1 / 2-C K O$ mice had normal-appearing lungs and hearts relative to those of controls. (D-F) At P3, Pbx1/2-CKO mice had evidence of lung simplification compared with controls but continued to have normal-appearing heart morphology. (G-I) At P28, in the few surviving Pbx1/2-CKO mice, there was severe alveolar simplification and cardiac hypertrophy relative to controls. No consistent hemorrhaging of the heart was observed. (J) Echocardiographic measurements of the RV wall thickness (RVWT) and LV wall thickness (LVWT) revealed that RV hypertrophy was present in $P b x 1 / 2$-CKO mice relative to control mice at P10 ( $P=$ $0.03)$, while the left ventricle had normal thickness $(P=0.48)$. (K) The PAAT/PAET ratio was used as an estimate for the pulmonary artery pressure and RV function. (L) The PAAT/PAET ratio was similar in Pbx1/2-CKO mice and controls at P3 $(P=0.45)$ but significantly different at P7 (** $P=0.004), \mathrm{P} 10,\left(^{* * *} P=\right.$ $0.008)$, and P14 ( $\left.{ }^{\#} P=0.001\right)$. For all statistical analyses, 4 samples were included from each group. Comparisons were made using a Student's $t$ test. Data represent the mean \pm SEM. Scale bars: $200 \mu \mathrm{m}$.

(Supplemental Figure 2A) and had no change in alveolar surfactant C (SPC) expression (Supplemental Figure 1, I and J). At PO, the lungs and hearts of $P b x 1 / 2$-CKO mice resembled those of their littermate controls (Figure 2, A-C). Despite their normal appearance at birth, $P b x 1 / 2-C K O$ mice failed to thrive, had tachypnea, were underweight, and started to die during the second week of life, with only rare survivors beyond 3 weeks of age (Supplemental Figure 2, A and B). Histological sections of Pbx1/2-CKO lungs showed alveolar simplification that was first evident at $\mathrm{P}$, as quantified by mean linear intercept (MLI) analysis (Figure 2, D and E, and Supplemental Figure 2, C and D). While grossly normal at P3
(Figure 2F), the hearts of $\mathrm{Pbx1} / 2$-CKO mice became progressively enlarged, with profound hypertrophy evident in surviving mice at P28 (Figure 2I). The majority of Pbx1/2-CKO mice died between 2 and 3 weeks of age, with lung simplification, cardiac hypertrophy, and right atrial enlargement (Figure 2, G-I).

Pbx1/2-CKO mutants exhibit defective alveologenesis. To determine the cause of the lung simplification in Pbx1/2-CKO mice, we looked for evidence of changes in cell proliferation or cell death at P3, when simplification is first evident. Using 5-ethynyl-2'-deoxyuridine (EdU) to mark proliferating cells and cleaved caspase-3 to mark cells undergoing apoptosis, we found no change in the rate of 

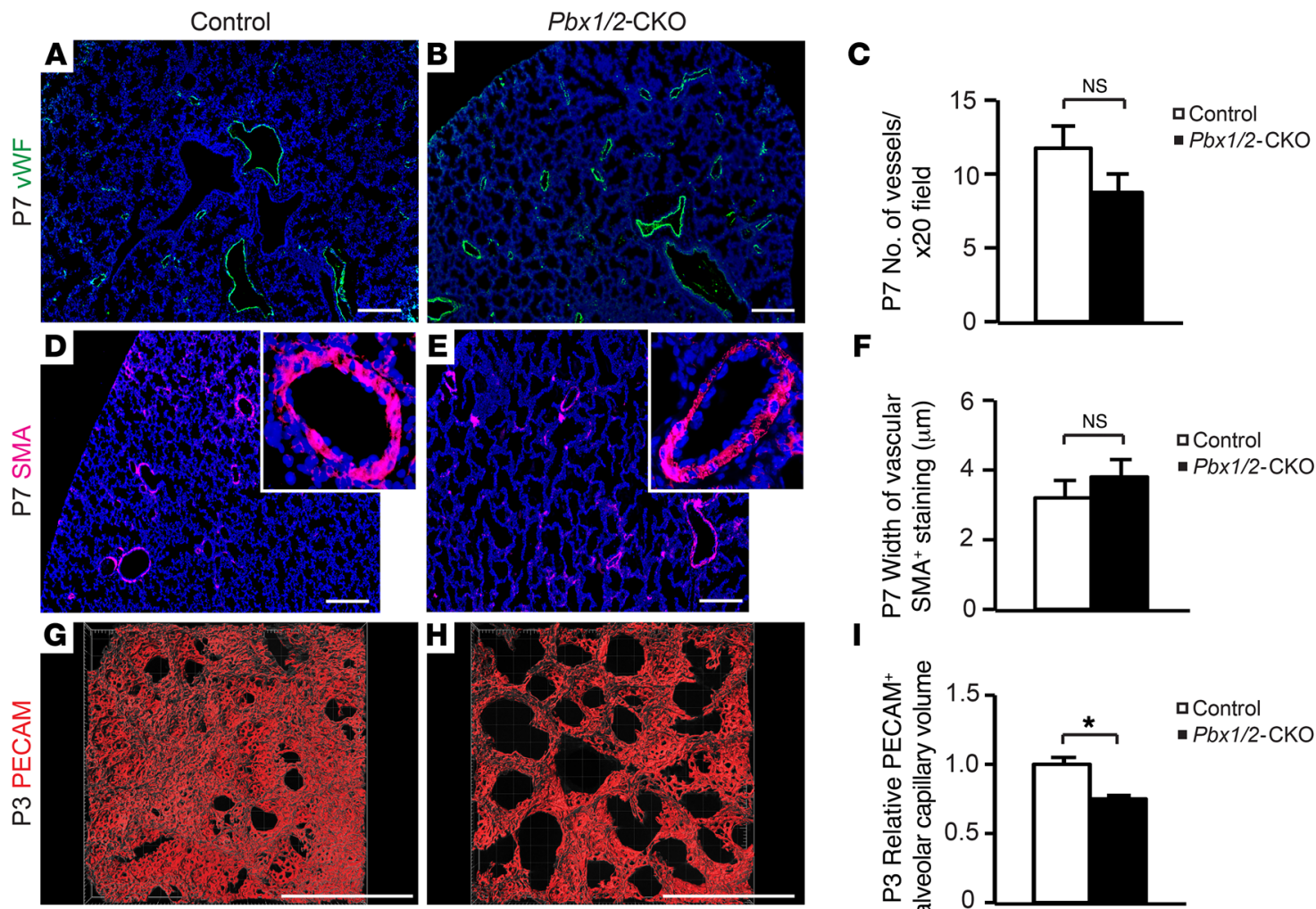

I
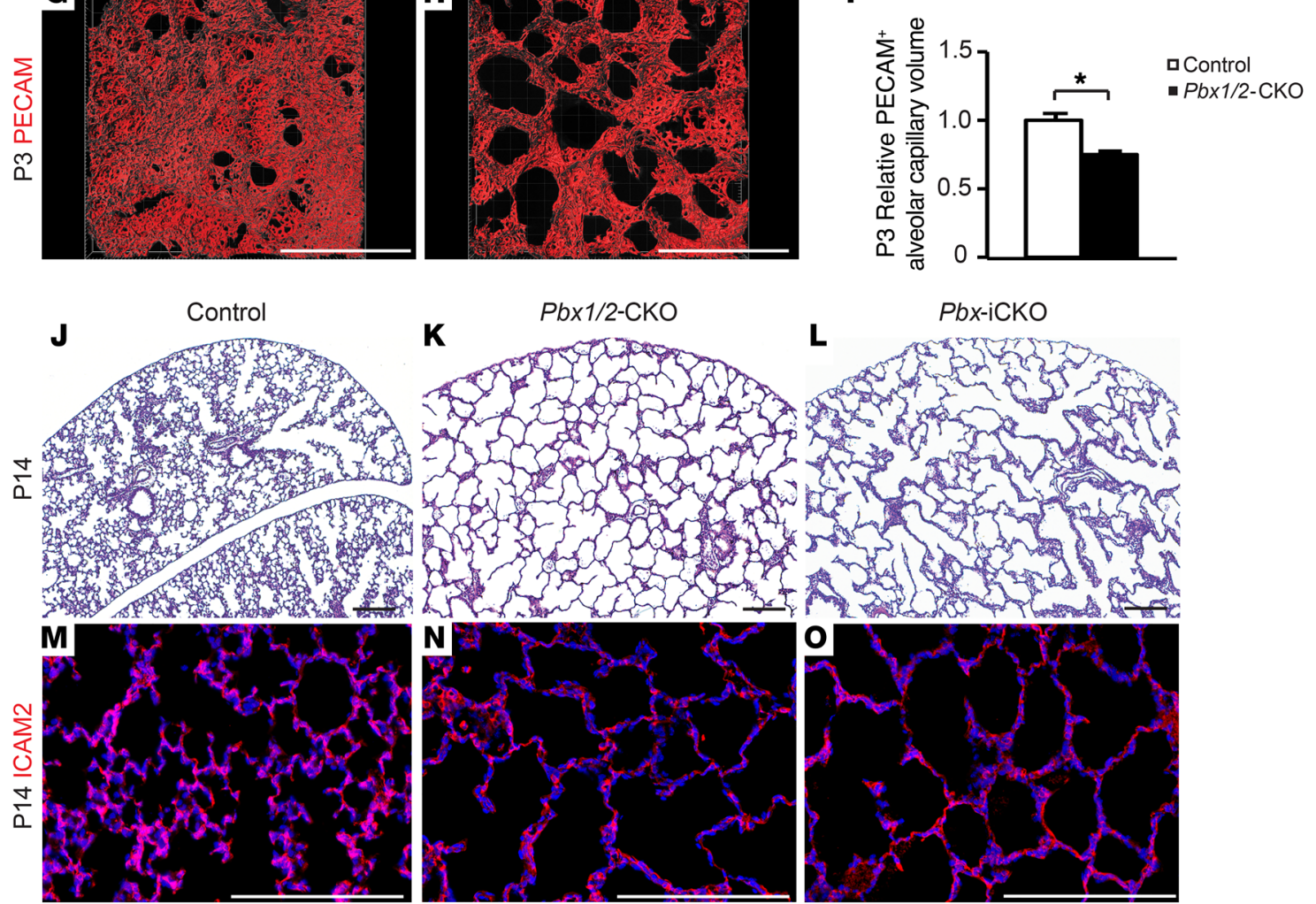

$\mathrm{Pbx}-\mathrm{iCKO}$

$\mathbf{L}$
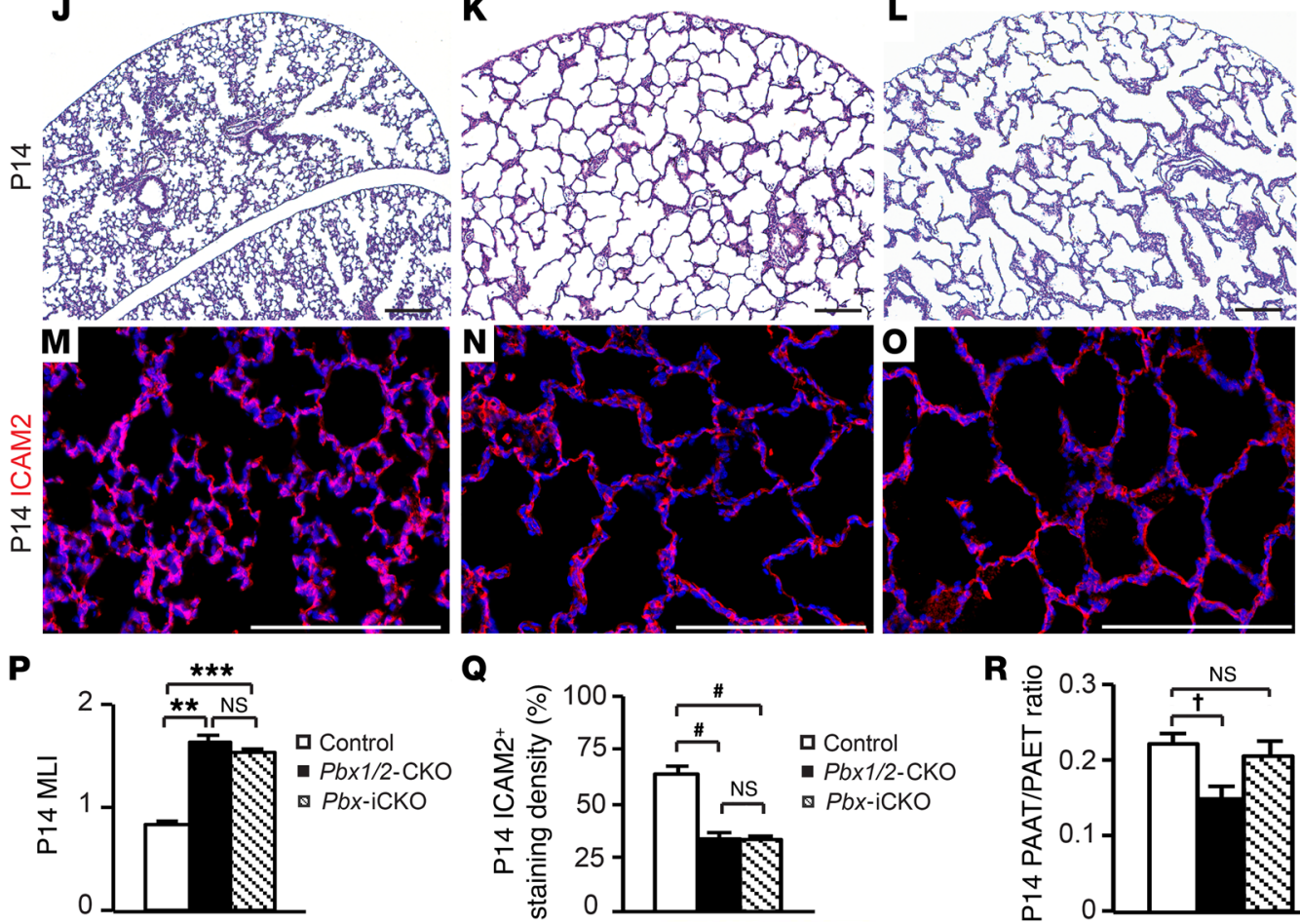

Q
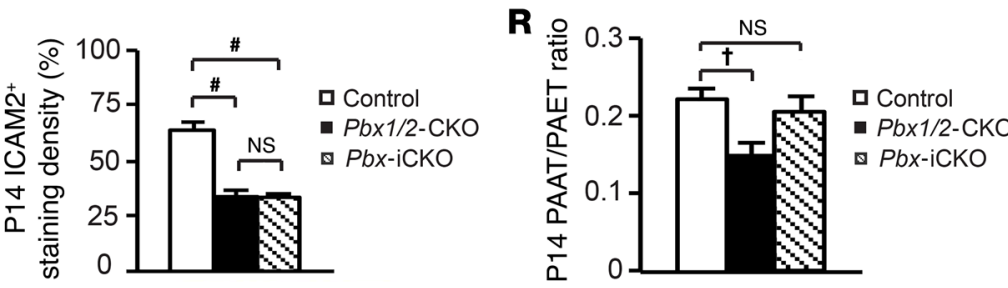

S VSM relaxation

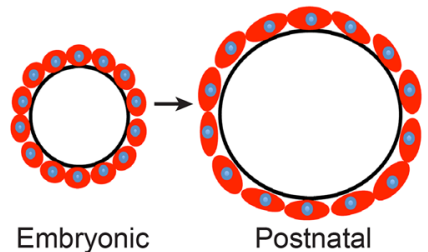

T

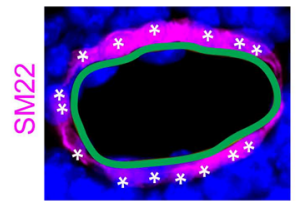

Smooth muscle cell size $=$ Vessel circumference -
SM22 nuclei number
$\mathbf{U}$

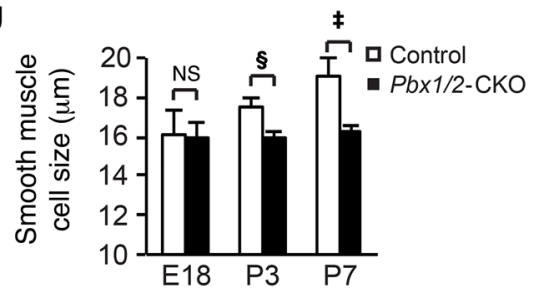


Figure 3. PH in Pbx1/2-CKO mice is associated with failed VSM relaxation after birth. (A-C) At P7, when hypertension was apparent, vWF staining of proximal vascular endothelial cells showed no significant difference in vessel numbers $(P=0.19)$. (D-F) At P7, SMA staining revealed no change in pattern and no smooth muscle hypertrophy $(P=0.44)$. (G-I) At P3, 3D reconstruction and quantification of PECAM-positive alveolar capillaries showed reduced PECAM density and volume ( $\left.{ }^{*} P=0.02\right)$. $(\mathrm{J}-\mathrm{L})$ At P14, H\&E staining showed alveolar simplification in $P b \times 1 / 2-C K O$ and $\mathrm{Pbx}$-iCKO mice relative to controls. $\mathrm{Tbx} 4^{\mathrm{Cre}} \mathrm{Pbx}^{+/ f l} \mathrm{Pbx}^{-/-}$and $\mathrm{Tbx} 4-\mathrm{rt}$ -

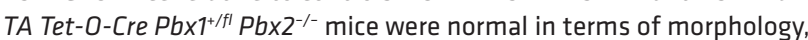
physiology, and capillary quantification and were used as a combined control. (P) MLI analysis revealed significant alveolar simplification in Pbx1/2-CKO $\left({ }^{* *} P<0.0001\right)$ and $P b x$-iCKO mice $\left({ }^{* *} P=0.0002\right)$ relative to controls ( $n=3$ in each group). $P b x 1 / 2-C K O$ and $P b x$-iCKO mice had similar alveolar simplification $(P=0.26)$. ( $\mathbf{M}-\mathbf{O}$ and $\mathbf{Q})$ ICAM2 staining shows reduced capillary endothelial density in $P b \times 1 / 2-C K O$ and $P b x$-iCKO mice ( $P=0.0007$ ) relative to controls ( $n=3$ in each group). Capillary density was similar in $P b x 1 / 2$-CKO and $P b x$-iCKO mice $(P>0.99)$. (R) The PAAT/ PAET ratio was reduced in $P b x 1 / 2$-CKO mice $\left({ }^{\dagger} P=0.007\right)$ but not in $P b x-$ iCKO mice $(P=0.82)$ compared with controls. (S) Illustration showing that VSM cells elongate after birth. (T) To estimate the average VSM cell size, the vessel perimeter was divided by the number of SM22-stained VSM cell nuclei. Asterisks indicate nuclei of SM22-positive cells. (U) Pbx1/2-CKO VSM cells were similar in size to control VSM cells prior to birth (E18, $P=$ 0.92 ) but did not increase in size after birth compared with controls ( $P 3$, $\left.{ }^{\$} P=0.02 ; \mathrm{P} 7,{ }^{\ddagger} P=0.04\right)$. For all statistical analyses, except MLI and ICAM2 staining density, 4 samples were included from each group. Comparisons between 2 groups were made using a Student's $t$ test or 1-way ANOVA for comparisons among 3 groups. Data represent the mean \pm SEM. Scale bars: $200 \mu \mathrm{m}$. Original magnification, $\times \mathbf{4 0}$ ( $\mathbf{D}$ and $\mathbf{E}$, insets).

cell proliferation or cell death (Supplemental Figure 3, A-F). Previous data from us and others showed that proper organization of the extracellular matrix, especially the Elastin matrix, is essential for normal alveolar septal formation (31-33). To determine whether loss of $\mathrm{PBX} 1 / 2$ is associated with defective extracellular matrix organization, we assayed for Elastin patterning using immunofluorescence staining of vibratome sections of the alveolar regions, confocal imaging, and 3D reconstruction. At the height of septum formation at P7, instead of a well-defined network of Elastin fibers as observed in the controls, the Elastin network in the Pbx1/2CKO lungs was diffuse and disorganized (Supplemental Figure 3, $G$ and $H)$. To determine the cause of this Elastin disorganization, we performed a gene expression array experiment and found that loss of PBX1/2 transcriptional regulation resulted in an increased expression of genes that promote formation, processing, and organization of the extracellular matrix, while the expression of genes required for cell-matrix interactions was decreased (Supplemental Figure 3I). These data suggest that PBX functions in alveologenesis through the transcriptional regulation of key genes that control the balance of Elastin matrix production, processing, and organization.

$\mathrm{Pbx1/2-CKO}$ mice exhibit $\mathrm{PH}$. To determine the progression of cardiac hypertrophy in $P b x 1 / 2-\mathrm{CKO}$ mice, we used transthoracic echocardiography to analyze $P b x 1 / 2-C K O$ and control mice. Measurements were made longitudinally at P3, P7, P10, and P14. To investigate the pattern of cardiac hypertrophy, we assayed for right ventricular (RV) and left ventricular (LV) wall thickness normalized to body weight. We found that the right ventricle of Pbx1/2-CKO mice was hypertrophied by P10 compared with that observed in control mice, while the left ventricle was similar in thickness at that stage (Figure 2J).
To determine the pulmonary vascular resistance (PVR), we attempted direct measurement of the $\mathrm{RV}$ pressure at early postnatal stages before death in $P b x 1 / 2-C K O$ mice. While we were able to obtain consistent measurements in the control mice, the $\mathrm{Pbx1} / 2$-CKO mice were too fragile and died during this procedure. Thus, we used transthoracic echocardiography to estimate the PVR. We calculated the ratio of the pulmonary artery acceleration time (PAAT) to the pulmonary artery ejection time (PAET) that is commonly used as a proxy for pulmonary artery pressure and RV function (Figure 2K) $(34,35)$. Using this approach, we found that control mice had a clear increase in the PAAT/PAET ratio between P3 and P7 and a more gradual increase between P7 and P14, indicating the expected reduction in pulmonary artery pressure after birth (Figure 2L). In contrast, $P b x 1 / 2-C K O$ mice had a gradual decline in the PAAT/PAET ratio after P3, suggesting that the pulmonary artery pressure did not decrease normally and that the right heart function may worsen with age (Figure 2L). The combination of findings including the decreased PAAT/PAET ratio and $\mathrm{RV}$ hypertrophy (Figure 2, J and I) is consistent with the presence of $\mathrm{PH}$ in the $\mathrm{Pbx} 1 / 2$-CKO mice. As $\mathrm{PH}$ is a major cause of lethality in patients with $\mathrm{CDH}$, the $P b x$-mutant mouse represents the first $\mathrm{CDH}$ model to our knowledge to mimic this key aspect of the disease, independent of the diaphragmatic defect.

Pbx1/2-CKO mutants develop $P H$ as a result of failed pulmonary VSM relaxation after birth. To determine the mechanism underlying the severe PH observed in Pbx1/2-CKO mutants, we investigated possible causes including decreased lung vessel number and size, VSM hypertrophy, and ectopic placement of VSM on distal pulmonary arterioles (36-38). At P7, when $\mathrm{PH}$ was detected by the PAAT/PAET ratio, we detected no statistically significant reduction in the number of vessels outlined by vWF staining (Figure 3, $\mathrm{A}-\mathrm{C})$. Similarly, we observed no difference in the pattern of vascular smooth muscle actin (SMA) staining (Figure 3, D and E) and no quantitative difference in the width of the VSM layer (Figure 3F), suggesting an absence of ectopic or increased VSM.

The alveolar simplification evident by P3 (Figure 2, D and E, and Supplemental Figure 2D) was accompanied by decreased capillary density (Figure 3, G and $\mathrm{H}$ ) and loss of capillary volume (Figure 3I). To determine whether this decrease in capillary volume is sufficient to cause $\mathrm{PH}$, we used doxycycline to induce Tbx4-rtTA TetO-Cre-mediated deletion of $\mathrm{Pbxl} / 2$ at E17 (referred to hereafter as $P b x$-iCKO mice), which resulted in loss of PBX1 expression in the distal mesenchyme but not in the proximal VSM (Supplemental Figure 4, A-F) (39). As was observed in Pbx1/2-CKO mice, the $P b x$-iCKO mutants had lung simplification and decreased capillary density relative to controls (Figure 3, J-Q). Interestingly, in contrast to the $P b x 1 / 2-C K O$ mice, which rarely survived beyond weaning, the $P b x$-iCKO mice were healthy at weaning and survived normally into adulthood, with no apparent increase in mortality (Supplemental Figure 4G). Using transthoracic echocardiography, we found that, despite alveolar simplification and decreased capillary density, $P b x$-iCKO mice, unlike $P b x 1 / 2$-CKO mice, showed no evidence of $\mathrm{PH}$, suggesting that decreased capillary density was not sufficient to cause PH in the Pbx mutants (Figure 3R).

Prior to birth, normal pulmonary VSM cells are contracted to limit blood flow through the lungs. After birth, VSM cells relax and elongate to allow for increased pulmonary blood flow (Figure 3S). 
A

VSM contraction and relaxation pathway

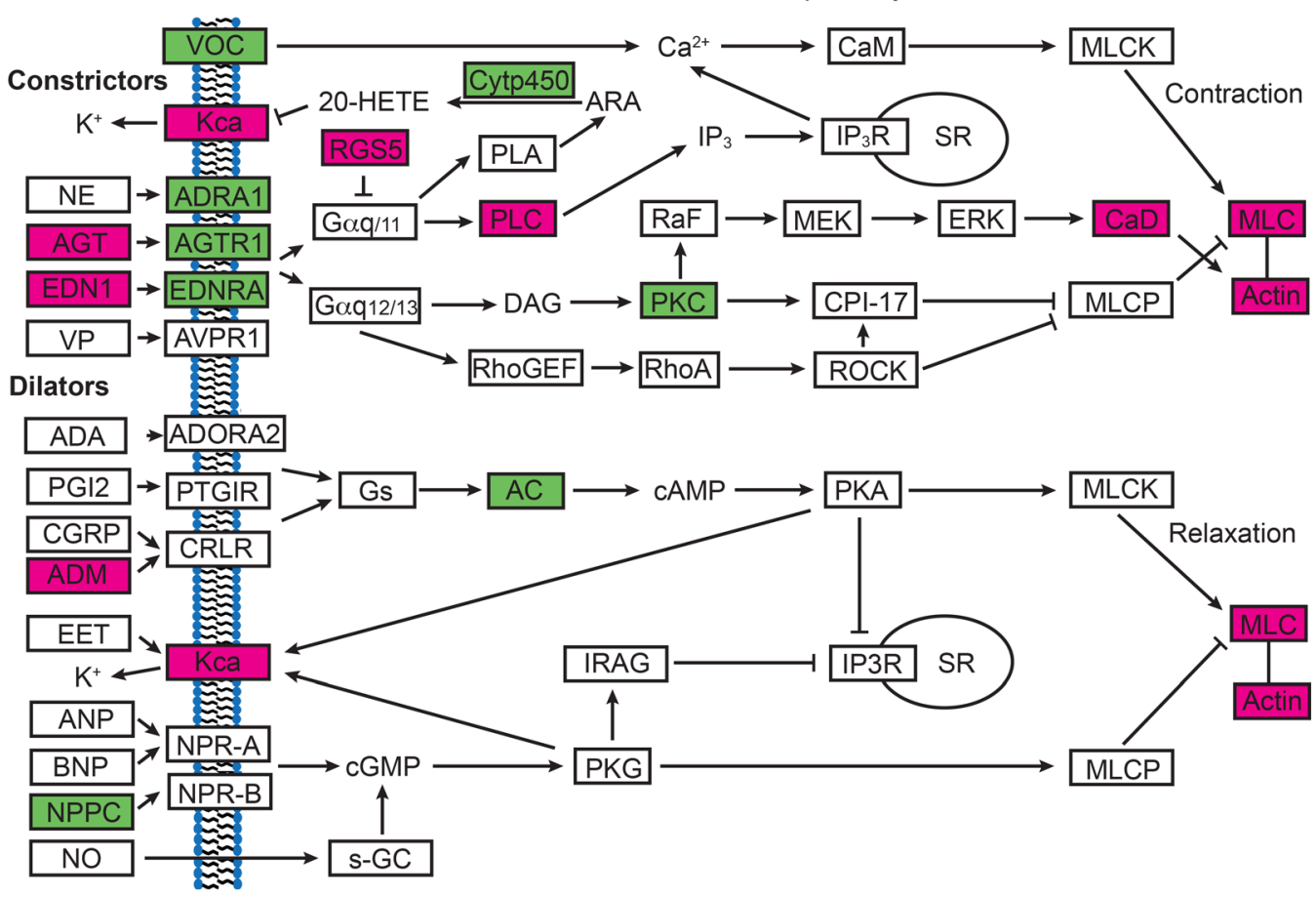

B Genes that regulate VSM tone

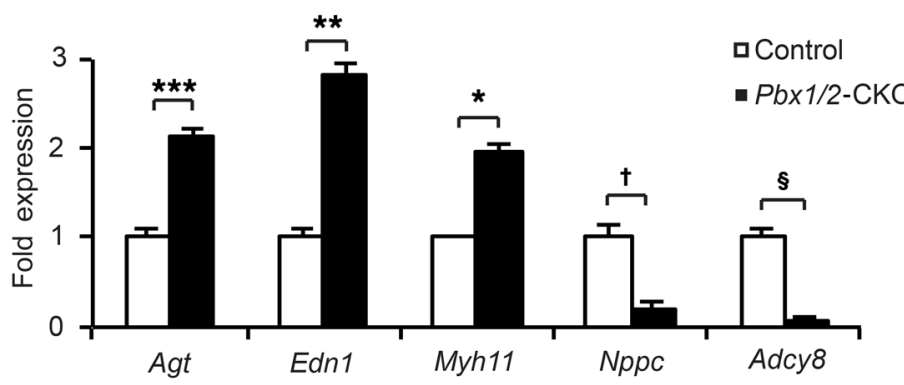

Figure 4. Pbx1/2 deletion affects multiple genes that control VSM contraction and relaxation. (A) Microarray gene expression analysis indicated that multiple genes that regulate VSM contraction and relaxation are upregulated (magenta) or downregulated (green) due to loss of PBX1/2 (modified from the Kyoto Encyclopedia of Genes and Genomes [KEGG] pathway map04270; http://www.genome.jp/dbget-bin/www_bget?pathway+hsa04270). (B) qRT-PCR of VSM contraction genes indicated increased expression of genes that promote contraction: Agt $\left({ }^{* * *} P=0.0006\right)$, Edn1 ( $\left.{ }^{* *} P=0.0005\right)$, Myh11 ( $\left.{ }^{*} P=0.0008\right)$, and decreased expression of genes that promote relaxation: $N p p c\left({ }^{\dagger} P=0.007\right)$ and $A d c y 8(\$ P=0.002)$. For all statistical analyses, 4 samples were included from each group. Comparisons were made using a Student's $t$ test. Data represent the mean \pm SEM.

Patients with $\mathrm{CDH}$ often have constricted pulmonary vessels that do not relax normally after birth $(8-11,40)$. We hypothesized that in the $P b x$-mutant model of $\mathrm{CDH}$, deletion of $P b x 1 / 2$ might impair the normal VSM relaxation process. To test this, we measured the circumference of lung vessels and divided this by the number of SM22-stained VSM cells surrounding each vessel to generate the average size of individual smooth muscle cells (Figure 3T). We found that prior to birth, the Pbx1/2-CKO mice had VSM cells that were similar in size compared with those of their littermate controls (Figure 3U). After birth, in control mice, we found that the VSM cells elongated from E18 to P3 and further elongated out to P7. In contrast, the VSM cells of Pbx1/2-CKO mice remained contracted, with no significant change in cell size from E18 to P3 or P7, and were statistically different when compared with controls at
P3 and P7 (Figure 3U). These results indicate that in Pbx1/2-CKO mutants, VSM cells do not relax after birth, resulting in persistent constriction of the pulmonary vessels.

Mesenchymal deletion of $\mathrm{Pbx} 1 / 2$ alters the expression of multiple genes involved in vascular contraction. To determine the molecular basis for the impact of loss of $P b x$, we performed unbiased transcriptome analysis of whole lungs from $\mathrm{Pbx} 1 / 2$-CKO and littermate control mice at E14, P0, and P3 and found that, among the differentially expressed transcripts, there was a significant enrichment of genes that regulate VSM contraction (Figure 4A and Supplemental Tables 1-3). Focusing on this class of genes, quantitative real-time PCR (qRT-PCR) analysis at P3, prior to overt $\mathrm{PH}$, showed that several genes that promote VSM contraction (endothelin-1 $[E d n 1]$, angiotensinogen $[A g t]$, and smooth 
A PAAT/PAET ratio after captopril or BQ-123

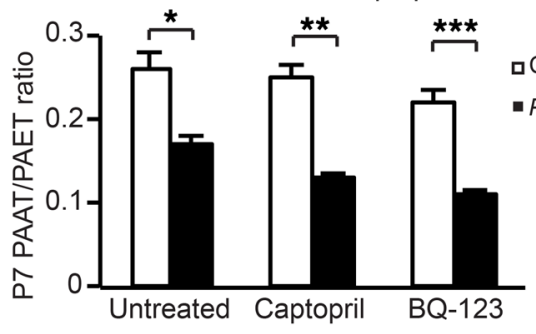

B Acute response to $B Q-123$

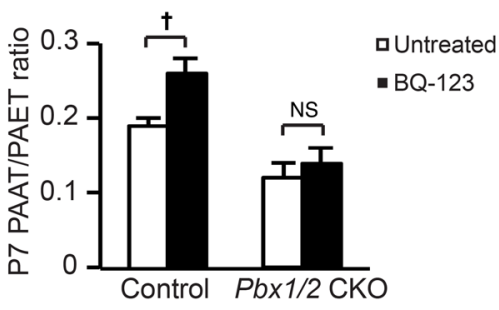

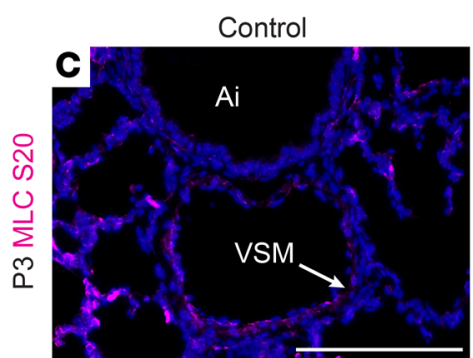

$\mathrm{Pbx} 1 / 2$ CKO PBS

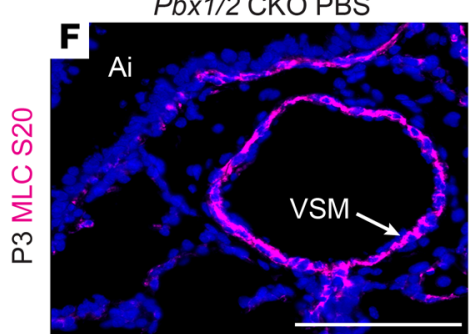

I
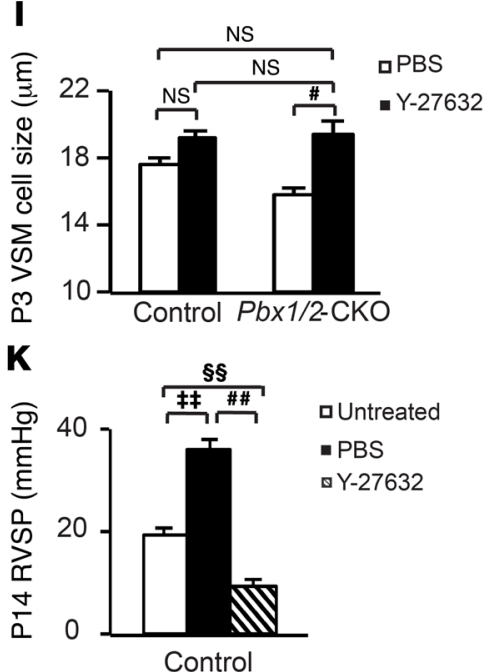

Control

- $P b \times 1 / 2-C K O$

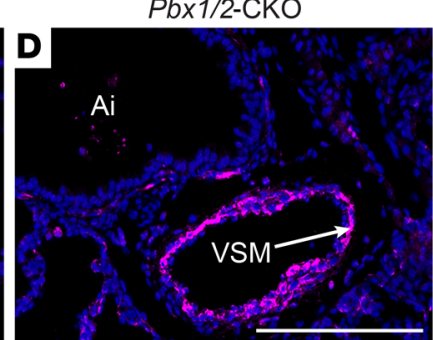

$\mathrm{Pbx} 1 / 2-\mathrm{CKO}$ Y-27632

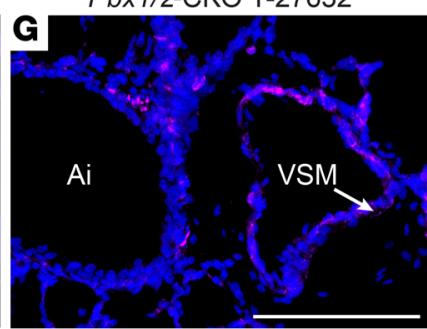

$\mathbf{J}$

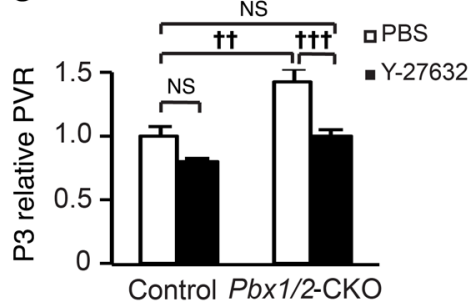

$\mathbf{L}$

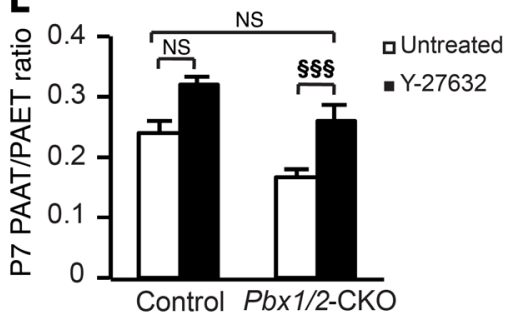

Figure 5. Abnormal VSM contraction in Pbx1/2-CKO mice is reversed by treatment with a Rho-kinase inhibitor. (A) Treatment of P0 to P7 mice with captopril or the endothelin receptor-A antagonist BQ-123 did not reduce the PVR of $P b \times 1 / 2-C K O$ mice compared with controls (untreated, ${ }^{*} P=0.004$; captopril-treated, ${ }^{* *} P=0.003$; BQ-123-treated, $\left.{ }^{* *} P=0.0004\right)$. (B) Acute treatment with $\mathrm{BQ}-123$ at $\mathrm{P} 7$ increased the PAAT/PAET ratio in control $\left({ }^{+} P=0.03\right)$ but not $P b \times 1 / 2$-CKO $(P=0.80)$ mice. (C-E) Phosphorylated MLC-S20 staining was increased in VSM cells from $P b \times 1 / 2-C K O$ mice $(\$ P=0.013)$. (F-H) Lung perfusion with the Rho-kinase inhibitor Y-27632 decreased MLC-S20 staining compared with PBS in $P b x 1 / 2$-CKO mice $\left({ }^{\ddagger} P=0.03\right)$. (I) Y-27632 perfusion at $\mathrm{P3}$ increased VSM cell size in $P b x 1 / 2-$ CKO $\left({ }^{\#} P=0.001\right)$ but not control $(P=0.14)$ mice. VSM cell size was similar following $Y-27632$ perfusion in control and $P b \times 1 / 2$-CKO lungs $(P=0.97)$. VSM cell size was similar between $Y$-27632-treated $P b \times 1 / 2$-CKO and PBS-treated control $(P=0.07)$ mice. $(J)$ PVR computed from the histological small artery size revealed increased resistance in $P b x 1 / 2$-CKO mice relative to controls $\left({ }^{\dagger+} P=0.008\right)$. Perfusion with Y-27632 significantly decreased the PVR in Pbx1/2-CKO lungs ( ${ }^{\dagger+t} P=0.02$ ) but not in control lungs $(P=0.36)$. The PVR of Y-27632-treated $P$ bx $1 / 2$-CKO mice was similar to that of untreated controls $(P=0.96)$. (K) Measurement at P14 showed increased RVSP following central venous injection of PBS $\left({ }^{\ddagger} P=0.0006\right)$ and decreased RVSP following injection into lungs of $\mathrm{Y}-27632$ compared with the RVSP before treatment $(\$ \$ P=0.008)$ and after treatment with PBS (\#\# $P<0.0001)$. (L) Acute treatment with $Y-27632$ increased the PAAT/PAET ratio in Pbx1/2-CKO mice $(\$ \$ \$=0.04)$ but not in controls $(P=0.08)$ and restored the PAAT/PAET ratio in Pbx1/2-CKO mice to that of the untreated controls $(P=0.22)$. For all statistical analyses, 4 samples were included from each group. Statistical analysis was performed using a Student's $t$ test for comparisons between 2 groups, 1-way ANOVA with Tukey's method for comparisons among 3 groups, or 2-way ANOVA with Tukey's method for comparisons between 2 groups under 2 or more conditions. Data are presented as the mean \pm SEM. Scale bars: $200 \mu \mathrm{m}$. 


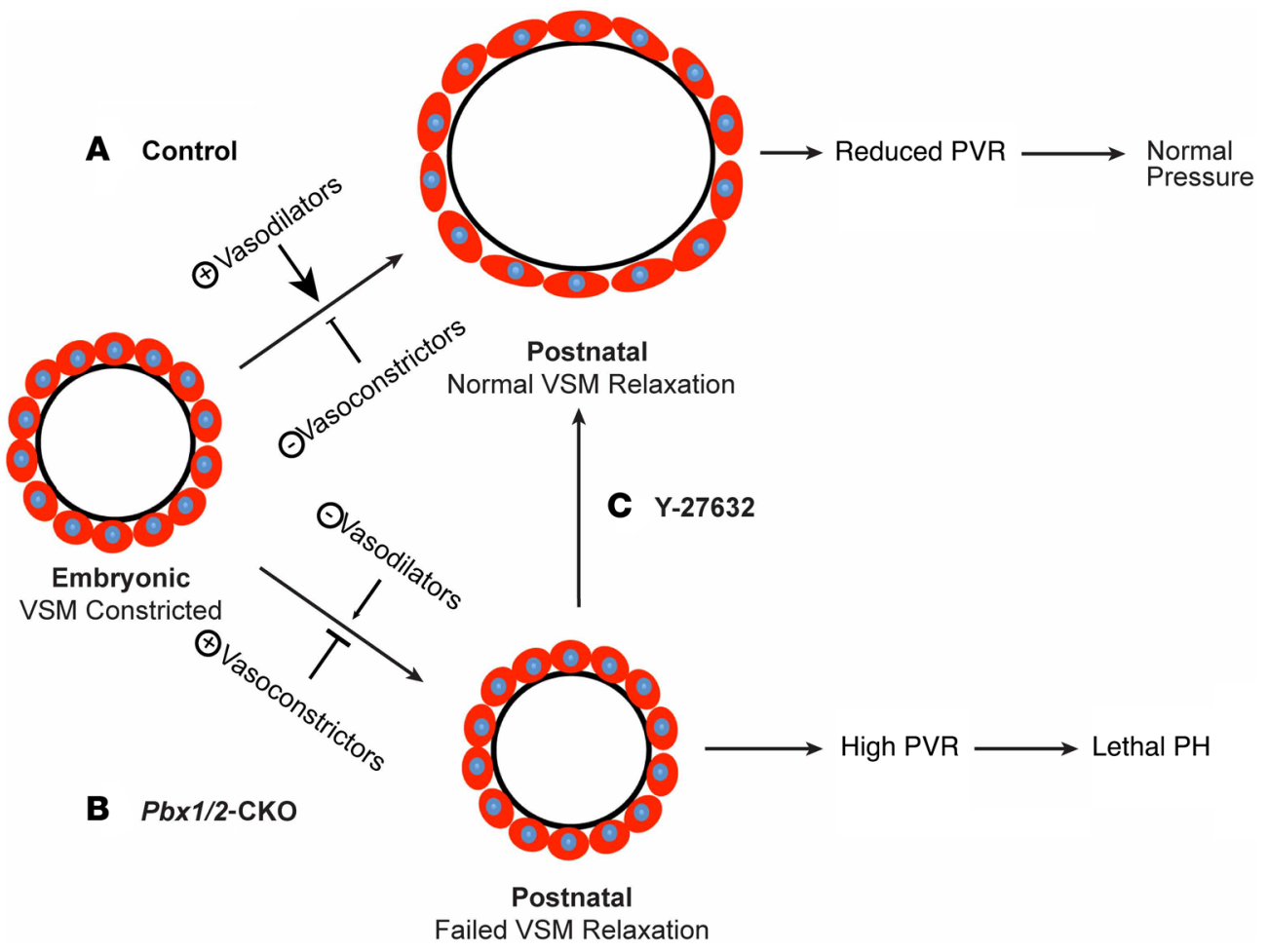

Figure 6. PBX plays a central role in perinatal pulmonary VSM relaxation. (A) Normal perinatal pulmonary VSM relaxation is the result of increased expression of factors that promote vasodilation and of decreased expression of factors that promote vasoconstriction. (B) Loss of PBX results in the failure of normal transition, with decreased expression of vasodilators and increased expression of vasoconstrictors causing high PVR and lethal PH. (c) Treatment with $\mathrm{Y}-27632$ restores pulmonary VSM relaxation and reduces $\mathrm{PH}$. muscle myosin [Myh11]) were upregulated, whereas genes that promote VSM relaxation (natriuretic peptide C $[N p p c]$ and adenylate cyclase $8[A d c y 8]$ ) were downregulated in mutants (Figure 4B). Interestingly, both Edn1 and Agt contain conserved PBX consensus-binding elements within the transcriptional regulatory regions of these genes (data not shown). No gene in the NO pathway was significantly altered (data not shown). These findings suggest that PBX plays a critical role in regulating the precise balance of factors that control vasoconstriction and vasodilation during the perinatal and early neonatal periods.

Pulmonary VSM contraction and $\mathrm{PH}$ in $\mathrm{Pbx1} / 2-\mathrm{CKO}$ mice are attenuated by downstream Rho-kinase inhibition. Infants with $\mathrm{CDH}$ and $\mathrm{PH}$ do not respond consistently to treatment with pulmonary vasodilating medications $(8-11,40)$. Identifying the basis of this variation has been a major challenge in $\mathrm{CDH}$ treatment. We sought to determine whether treatment with pharmacological agents that target the misregulated genes in $\mathrm{Pbx} 1 / 2-\mathrm{CKO}$ mice could rescue their lethal $\mathrm{PH}$. To reverse the upstream increase in $E d n 1$ and $A g t$ expression, we treated $\mathrm{Pbx1} / 2-\mathrm{CKO}$ and control mice from PO to P7 with an endothelin receptor A antagonist (BQ-123) or an angiotensin-converting enzyme inhibitor (captopril) and measured the PAAT/PAET ratio at P7 (Supplemental Figure 5A). We found that neither treatment was effective in normalizing the PAAT/PAET ratio of $\mathrm{Pbx1} / 2-\mathrm{CKO}$ mice compared with controls (Figure 5A). Because BQ-123 has a short half-life, we also investigated changes in PVR after acute treatment of $\mathrm{Pbx1/2-CKO}$ mice and littermate controls. We used transthoracic echocardiography to measure the PAAT/PAET ratio in P7 Pbx1/2-CKO and control mice. These mice were then treated with BQ-123, and the PAAT/ PAET ratio measurement was repeated 2 hours after treatment (Supplemental Figure 5B). Using this approach, we found that, although BQ-123 induced an increase in the PAAT/PAET ratio in control mice, we did not observe a statistically significant increase in $\mathrm{Pbx1} / 2-\mathrm{CKO}$ mice (Figure 5B). These data suggest that blocking signals that promote vasoconstriction at the level of cell-surface receptors is not sufficient to reverse $\mathrm{PH}$ in Pbx1/2-CKO mice, possibly because of PBX regulation of other factors that act epistatically to these signals.

Because several downstream factors that act within the VSM cells to promote constriction are altered, we hypothesized that a common downstream effect could be an imbalance of myosin light chain kinase (MLCK) and myosin light chain phosphatase (MLCP), causing increased phosphorylation of myosin light chain (MLC) and subsequent increased VSM contraction. Using phosphorylated smooth muscle MLC (Ser20) (MLC-S20) as an indicator, we found that $\mathrm{Pbx1} / 2-\mathrm{CKO}$ mice had increased MLC-S20 staining in VSM compared with that in controls, supporting this hypothesis (Figure 5, C-E).

We then tested whether an agent that acts directly on the balance of MLCK/MLCP activity, such as the Rho-kinase inhibitor Y-27632, might be effective at inducing VSM relaxation and reducing $\mathrm{PH}$ in $\mathrm{Pbx1} / 2-\mathrm{CKO}$ mice. Lung vascular perfusion with Y-27632 resulted in decreased MLC-S20 staining of VSM in $\mathrm{Pbx1} / 2$-CKO mice at $\mathrm{P} 3$ compared with that observed in $\mathrm{Pbx1} / 2$ CKO mice perfused with PBS (Figure 5, F-H). In addition, perfusion with Y-27632 resulted in increased VSM cell size in Pbx1/2CKO mice compared with VSM cell size in lungs perfused with PBS (Figure 5I). These data indicate that treatment with Y-27632 promotes VSM relaxation in $\mathrm{Pbx} 1 / 2$-CKO mice.

To estimate the effect of Y-27632-induced smooth muscle relaxation on relative PVR following treatment with Y-27632, we used a mathematical model to compute changes in resistance to blood flow through the lung based on the measured small artery 
size. The results showed that prior to treatment, $\mathrm{Pbx} 1 / 2-\mathrm{CKO}$ mice had increased PVR relative to controls (Figure 5J). Following lung perfusion with Y-27632, the model predicted a decrease in PVR in Pbx1/2-CKO mice but not in control mice (Figure 5J). Furthermore, treatment of $P b x 1 / 2-C K O$ mice with Y-27632 was estimated to reduce the PVR to that observed in PBS-treated control mice (Figure 5J).

To determine whether the prediction based on artery size reflects PVR in vivo, we measured the RV peak systolic pressure (RVSP) in control mice before and after treatment with Y-27632 (Figure 5K). Treatment with Y-27632 decreased the RVSP relative to baseline, however the effect was transient, lasting only 3 to 5 minutes (Figure $5 \mathrm{~K}$ ). To determine whether the Y-27632-induced increase in small artery size, the decrease in the predicted PVR, and the decrease in the RVSP translate to decreased $\mathrm{PH}$ in $\mathrm{Pbx1} / 2-\mathrm{CKO}$ mice in vivo, we measured the PAAT/PAET ratio of P7 Pbx1/2-CKO and control mice before and 2 hours after Y-27632 treatment (Supplemental Figure 5B). Treatment with Y-27632 led to an increase in the PAAT/ PAET ratio in $\mathrm{Pbx1} / 2-\mathrm{CKO}$ mice but not in controls (Figure $5 \mathrm{~L}$ ). Furthermore, the PAAT/PAET ratio in the treated $\mathrm{Pbx1} / 2-\mathrm{CKO}$ mice was similar to that in the untreated control mice. These data suggest, as predicted by both the change in VSM cell size and PVR modeling, that treatment with the Rho-kinase inhibitor was effective at normalizing the PVR in Pbx1/2-CKO mice to that of controls (Figure 5L). We note that prolonged treatment with i.p. injection of Y-27632 led to lethality in both control and mutant mice, possibly because of the pleiotropic effects of Rho-kinase inhibition, including that of systemic hypotension $(41,42)$. This suggests that targeted delivery and timed release of the agent to lung VSMs will be required to successfully prevent lethal $\mathrm{PH}$ in this genetic model.

\section{Discussion}

A drastic increase in pulmonary blood flow in the perinatal period is critical for survival at birth (43). This transition relies on relaxation of pulmonary VSMs. Multiple factors have been demonstrated to control this process, including both non-cell-autonomous, upstream mechanisms such as oxygen, NO signaling, and endothelin-1 (ET-1), as well as cell-autonomous, downstream mechanisms including cGMP and PDE5 (44-46). However, how these factors are coordinated in a short amount of time to allow the necessary abrupt increase in blood flow is not known. Here, we identify PBX transcription factors as a principal controller of this process in the lungs. PBX regulates the expression of both vasoconstrictors and vasodilators that culminates in the reduction of PVR. Failure of this reduction in $\mathrm{Pbx1} / 2-\mathrm{CKO}$ mutants resulted in a genetic model of lethal $\mathrm{PH}$ in mice (Figure 6).

In patients with $\mathrm{CDH}, \mathrm{PH}$ has been postulated to arise from several causes including mechanical compression by herniated organs, decreased vessel numbers, decreased capillary density, and decreased vessel size caused by smooth muscle hypertrophy or persistent smooth muscle contraction (6-9, 47-49). An improved understanding of the primary causes of $\mathrm{PH}$ in individual $\mathrm{CDH}$ patients will help direct effective treatment. Our approach has been to use a mouse model of $\mathrm{CDH}$ to determine the degree to which each of these factors contributes to the severity of $\mathrm{PH}$.
All infants with $\mathrm{CDH}$ have some degree of mechanical compression of the lung that contributes to $\mathrm{PH}$ after birth. This direct, causal relationship has been challenged, for example, in cases of congenital pulmonary airway malformation (CPAM), in which mechanical compression of the lung does not lead to elevated PVR (12). Our findings from the lung mesenchymal deletion of $P b x 1 / 2$ demonstrate for the first time to our knowledge that molecular defects intrinsic to the lung are sufficient to cause $\mathrm{PH}$ independently of the diaphragmatic defect and mechanical compression.

Abnormal muscularization of distal, intra-acinar arteries and failure of perinatal pulmonary VSM relaxation are both common features in $\mathrm{CDH}$ patients with severe $\mathrm{PH}$ (37). In the $\mathrm{Pbx1} / 2$ deletion model of $\mathrm{CDH}$, there is no ectopic VSM on distal arterioles, so this model cannot be used to assess the impact of excess smooth muscle. Instead, our data demonstrating failed smooth muscle cell expansion and increased MLC phosphorylation indicate persistent smooth muscle contraction. Reversal of these defects relieved VSM contraction and normalized the PAAT/PAET ratio, suggesting that smooth muscle contraction is a major contributor to lethal PH in this model.

In patients with $\mathrm{CDH}$, direct measurement of PVR can be done in the cardiac catheterization laboratory. However, because of the invasive nature of this approach, patients are routinely monitored by echocardiography to estimate the pulmonary vascular pressures and response to $\mathrm{PH}$ medications. In this study, while we were able to obtain a direct measurement of RV pressure in control mice, none of the $P b x 1 / 2-C K O$ mice survived the procedure to yield data. In comparison, transthoracic echocardiography provided consistent and longitudinal data in both mutant and control mice. Changes in the PAAT/PAET ratio measured by echocardiography have been shown to sensitively reflect fluctuations in pulmonary artery pressure, PVR, and RV function $(34,35)$. In this study, we were able to use transthoracic echocardiography and the PAAT/PAET ratio to link physiology to cellular mechanisms. Our data suggest that the persistence of smooth muscle contraction after birth significantly changes the PAAT/PAET ratio and is the primary contributor to lethal $\mathrm{PH}$ in $\mathrm{Pbx}$ mutants.

The abnormal expression of genes that cause persistent VSM contraction in $\mathrm{Pbx} 1 / 2-\mathrm{CKO}$ mutant mice is consistent with reports on patients with CDH. Several proteins that control VSM tone, including ET-1 and prostacyclin (PGI2), have been found to be present in abnormal concentrations in patients with $\mathrm{CDH}(49-51)$. The documented abnormal expression of these factors has been associated with more severe $\mathrm{PH}$ and worse clinical outcome in these patients. Interestingly, even though the gene expression and physiological abnormalities responsible for $\mathrm{PH}$ in $\mathrm{Pbx}$-mutant mice are not evident until after birth, the level and pattern of PBX1 expression does not appear to change in the late embryonic and early postnatal period (Figure 1). These data suggest that the developmental and physiological roles played by PBX in the lungs are modulated by its posttranslational modification, transcriptional partners, and/or downstream mediators that act in a chronological and cell-type-specific manner.

Another question that remains a key challenge in $\mathrm{CDH}$ is why some patients with $\mathrm{PH}$ do not respond to vasodilating medications. Many of the available medications to treat $\mathrm{PH}$ address 
upstream vasorelaxation either via receptor-mediated effects (oxygen, inhaled NO [iNO], prostacyclin analogs, and endothelin receptor antagonists), or at the cyclic GMP or cyclic AMP (cAMP) second messenger level (inhibitors of phosphodiesterase 5 and 3). We show here that loss of $P b \times 1 / 2$ is associated with misregulation of multiple factors that culminate downstream to control VSM tone. Even though loss of PBX transcriptional repression resulted in increased expression of $E d n 1$ and $A g t$, targeting these upstream factors was not effective at reducing $\mathrm{PH}$. Our data suggest that $\mathrm{PBX} 1 / 2$ transcriptional regulation is required for maintaining the balance of gene expression necessary for normal postnatal pulmonary VSM relaxation. Targeting the common downstream effectors, such as Rho-kinase, was more effective at reversing smooth muscle contraction and reducing $\mathrm{PH}$. It should be noted that the serial systemic administration of the Rho-kinase inhibitor resulted in lethality in both control and $\mathrm{Pbx} 1 / 2-\mathrm{CKO}$ mice, probably due to the systemic hypotension that has been reported previously following systemic treatment $(41,42)$. Targeted and time-restricted delivery of agents like these to the pulmonary VSM is critical to safely take advantage of their therapeutic benefit. Nevertheless, these results raise the possibility that some patients with $\mathrm{CDH}$ may have defects in the downstream regulators of VSM contraction, which would explain the observed lack of response to drugs that target upstream factors. In these patients, targeting the downstream regulators may prove more effective.

Genetic evaluation of patients with $\mathrm{CDH}$ is now standard, with fetal microarray analysis being offered to families. Whole-exome and whole-genome sequencing is being performed on an increasing number of patient and family trios and has led to the identification of a number of single-gene variants that are associated with $\mathrm{CDH}(52-54)$. Our research highlights the opportunity to link individual gene mutations to prognosis and indications for specific therapeutic approaches. As whole-genome sequencing becomes more available and the diverse mechanisms linked to individual mutations associated with $\mathrm{CDH}$ become clear, these will greatly improve the accuracy and resolution of prenatal testing offered to families. The data obtained will help to identify fetuses that have an increased risk of lethal $\mathrm{PH}$ and also provide clinicians with a targeted and effective treatment strategy.

\section{Methods}

Animal handling and genetics. All mice were bred on a mixed genetic background, and age-matched littermates were used for comparison.

$P b x 1$-floxed and Pbx2-null alleles were previously described (29, 55). Recombination of the conditional $P b x 1$ allele was achieved by crossing with the $T b x 4^{\text {Cre }}$ allele or Tbx4-rtTA TetO-Cre transgenes, as previously described $(28,39)$. Tbx4-rtTA was induced by feeding doxycycline food (Harlan Laboratories) to pregnant females starting on E17. Cre-positive, $P b x 1$-heterozygous, and Pbx2-null ( $\mathrm{Tb} x 4^{\mathrm{Cre}} \mathrm{Pbx} \mathrm{1}^{\mathrm{fl}+\mathrm{H}}$ $P b x 2^{4 / \Delta}$ ) littermates were used as controls.

Histology. Mice were euthanized and lungs were inflated with $4 \%$ paraformaldehyde (PFA) by gravity inflation with a pressure of $20 \mathrm{~cm}$ $\mathrm{H}_{2} \mathrm{O}$. Lungs were then prepared for paraffin $(8 \mu \mathrm{m})$, cryo- $(10 \mu \mathrm{m})$, or vibratome $(100 \mu \mathrm{m})$ sectioning. For each histological assessment, a minimum of 4 mice from each group were analyzed using matched sections from the apex, middle, and base of the lungs of each mouse.
MLI analysis. The MLI of control, Pbx1/2-CKO, and $P b x$-iCKO lungs was calculated using $\times 20 \mathrm{H} \& \mathrm{E}$-stained images. Three mice from each group were analyzed, with three images taken from sections of the apex, middle, and base of the lungs of each mouse. The MLI was calculated as previously described using a $12 \times 8,50-\mu \mathrm{m}$ grid (56). The average MLI was calculated for each mouse and then averaged within each group. The data are reported as the MLI \pm SEM, with statistical analysis performed using a Student's $t$ test for comparisons between 2 groups or 1-way ANOVA with Tukey's method for comparisons among 3 groups.

Immunofluorescence staining. The following primary antibodies were used for immunofluorescence: mouse anti-PBX1b, 1:100 (Santa Cruz Biotechnology; catalog sc-101852, clone 41.1); rabbit anti-SM22 $\alpha, 1: 200$ (Abcam; catalog ab14106); mouse anti-SMACy3, 1:200 (Sigma-Aldrich; catalog C6198, clone 1A4); rabbit antiVWF, 1:50 (Sigma-Aldrich; F3520); rat anti-PECAM (CD31), 1:100 (BD Biosciences; catalog 551262, clone MEC13.3); rabbit anti-ADRP, 1:100 (Abcam; catalog ab52356); rabbit anti-MLCS20, 1:100 (Abcam; catalog ab2480); rabbit anti-GFP, 1:100 (Synaptic Systems; catalog 132002); rabbit anti-PDGFR $\beta$, 1:100 (Cell Signaling Technology; catalog 3169P, clone 28E1); rat anti-ICAM2, 1:100 (BD Biosciences; catalog 553325, clone 3C4); rabbit anti-tropoelastin, 1:100 (Abcam; catalog ab21600); and rabbit anti-cleaved caspase-3, 1:100 (Cell Signaling Technology; catalog 9661S). The following secondary antibodies were used: goat anti-rabbit CY3, 1:200 (Jackson ImmunoResearch; catalog 111-165-144); goat anti-rabbit FITC, 1:200 (Jackson ImmunoResearch; catalog 111-095-144); goat anti-mouse CY3, 1:200 (Jackson ImmunoResearch; catalog 115-165-003); goat anti-rat CY3, 1:200 (Jackson ImmunoResearch; catalog 112-165-003); and goat antirabbit CY5, 1:200 (Jackson ImmunoResearch; catalog 111-175-144).

Cell proliferation and apoptosis assays. EdU was used to label newly synthesized DNA according to the protocol provided with the ClickiT EdU Alexa Fluor 488 Imaging Kit (Invitrogen, Thermo Fisher Scientific). Mice were injected with EdU 60 minutes prior to euthanasia and tissue collection. Immunostaining for cleaved caspase- 3 was used to label cells undergoing apoptosis. Paraffin-embedded lung sections $(8-\mu \mathrm{m})$ from the apex, middle, and base of equivalent lung regions from 4 control mice and $4 \mathrm{Pbx} 1 / 2-\mathrm{CKO}$ littermates were stained and imaged using a $\times 40$ objective. The rate of cell proliferation or cell death in the alveolar region of the lung was determined by dividing the number of EdU- or cleaved caspase-3-positive cells by the total number of nuclei stained by DAPI, respectively. Data are presented as the mean rate of cell proliferation (percentage of EdU-positive cells) or cell death (percentage of cleaved caspase-3-positive cells) \pm SEM, with statistical comparison made using a Student's $t$ test.

Transthoracic echocardiography. Transthoracic echocardiography was performed by the Cardiovascular Physiology Core Facility at the University of Wisconsin using a VisualSonics Vevo 770 ultrasonograph with a 40-MHz transducer (57).

End-diastolic and end-systolic RV and LV diameter as well as anterior wall and posterior wall thickness were measured online from M-mode images using the leading edge-to-leading edge convention. These measurements were normalized to mouse body weight. The data are reported as the average normalized RV and LV wall thickness, with statistical comparison made using a Student's $t$ test. As a proxy for PVR, we measured the PAAT/PAET ratio. PAAT and PAET were measured directly, and the PAAT/PAET ratio was calculated for each 
mouse $(34,35)$. The data are reported as the average PAAT/PAET ratio \pm SEM for each group, with statistical comparisons made using a Student's $t$ test for comparisons between 2 groups, 1-way ANOVA with Tukey's method for comparisons among 3 groups, or 2-way ANOVA with Tukey's method for comparisons between 2 groups under 2 or more conditions. Mice older than P7 were anesthetized with isoflurane during transthoracic echocardiography.

$3 D$ Elastin fiber staining and capillary volume measurement. Vibratome-cut sections (100- $\mu \mathrm{m})$ of equivalent alveolar regions from 4 control and $4 \mathrm{Pbx} 1 / 2$-CKO mice were stained by immunofluorescence for tropoelastin or PECAM and imaged using an LSM 510 confocal laser scanning microscope (Carl Zeiss) equipped with an argon laser (excitation $488 \mathrm{~nm}$ ) and a DPS laser (excitation $561 \mathrm{~nm}$ ). Serial optical sections ( $80 \mu \mathrm{m}$ total depth, $1 \mu \mathrm{m} Z$-step) were used to generate a surface rendering of the tropoelastin-stained Elastin fibers or the PECAM-stained alveolar capillaries of each vibratome section. Imaris software (Bitplane, Oxford Instruments) was used to calculate the volume of each PECAM surface rendering. The average PECAM volume was calculated for each mouse and then averaged within each group. The data are presented as the average PECAM volume normalized to the control samples \pm SEM, with statistical comparison made using a Student's $t$ test.

ICAM2-stained areapercentage. Paraffin-embedded sections (8- $\mu \mathrm{m})$ from the apex, middle, and base of lungs from 3 control, $3 \mathrm{Pbx} 1 / 2-\mathrm{CKO}$, and $3 \mathrm{Pbx}$-iCKO mice were stained by immunofluorescence for ICAM2 and imaged using a $\times 20$ objective. The percentage of area covered by ICAM2 staining was determined by counting pixels using Image J software (NIH). The average percentage of ICAM2-stained area was calculated for each mouse and then averaged within each group. The data are presented as the average percentage of ICAM2-stained area \pm SEM, with statistical comparisons made using 1-way ANOVA with Tukey's method to adjust for multiple comparisons.

Smooth muscle cell size analysis. Paraffin-embedded sections $(8-\mu \mathrm{m})$ from the apex, middle, and base of lungs from 4 control, 4 $\mathrm{Pb} x \mathrm{1} / 2$-CKO, and $4 \mathrm{Pbx}$-iCKO mice were stained for SM22 by immunofluorescence and imaged using a $\times 40$ objective (see Figure $3 \mathrm{~T}$ ). The inner circumference (green line) of each SM22-positive vessel was measured using ImageJ software. The nuclei of SM22-positive cells (indicated by asterisks) were counted. The circumference was then divided by the number of nuclei, resulting in an average SM22-positive VSM cell length per vessel. The average smooth muscle cell size was calculated for each mouse and then averaged within each group. The data are presented as the average smooth muscle cell size $(\mu \mathrm{m}) \pm$ SEM using a Student's $t$ test for comparisons between 2 groups or 2-way ANOVA with Tukey's method for comparisons between 2 groups under 2 or more conditions.

qRT-PCR and microarray analysis. All qRT-PCR experiments were conducted using 3 biological and 3 technical replicates. Lungs from $\mathrm{Pb} x \mathrm{1} / 2$-CKO and control mice were homogenized in TRIzol (Thermo Fisher Scientific), and RNA was isolated using an RNeasy Plus Mini Kit (QIAGEN). cDNA was generated using a Superscript III First-Strand Synthesis System (Invitrogen, Thermo Fisher Scientific). qRT-PCR was quantified using SYBR green (Applied Biosystems) and run on a LightCycler 480 (Roche). Gene expression was normalized to $\beta$-actin. The data are presented as the relative expression normalized to controls \pm SEM, with statistical comparison made using a Student's $t$ test. The primer sets used are listed in Supplemental Table 4.
For microarray, lungs from $3 \mathrm{Pbx} 1 / 2-\mathrm{CKO}$ or 3 control mice were pooled at each stage. RNA $(1 \mu \mathrm{g})$ was analyzed from each pooled sample at the University of Wisconsin - Madison Gene Expression Center using a Mouse Gene 1.0 Array (Affymetrix). GeneSifter software (Geospiza) was used to analyze differential gene expression. The gene expression microarray data are deposited in the NCBI's Gene Expression Omnibus (GEO) database (GEO GSE106399; https://www.ncbi. nlm.nih.gov/geo/query/acc.cgi?acc=GSE106399).

Drug treatments. Captopril (Sigma-Aldrich), BQ-123 (SigmaAldrich), and Y-27632 (Tocris) were used to treat $P b x 1 / 2-C K O$ and control mice by i.p. injection. Mice that did not receive drug treatment were given PBS by i.p. injection. For PO to P7 daily treatment (Supplemental Figure 5A), mice were treated once a day with PBS, captopril $(0.5 \mathrm{mg} / \mathrm{kg})$, or BQ-123 (10 mg/kg) and underwent transthoracic echocardiography on P7. For acute treatment (Supplemental Figure 5B), P7 mice were analyzed by transthoracic echocardiography, treated with BQ-123 (10 mg/kg), Y-27632 $(50 \mathrm{mg} / \mathrm{kg})$, or PBS and then reanalyzed by transthoracic echocardiography 2 hours after treatment. The average PAAT/PAET ratio was calculated for each group before and after treatment. The data are presented as the average PAAT/PAET ratio \pm SEM, with statistical comparison made using a Student's $t$ test.

For ex vivo Y-27632 treatment of control and Pbx1/2-CKO mice, the mice were euthanized and the right ventricle immediately perfused with Y-27632 $(10 \mu \mathrm{M})$ or PBS. Immediately following treatment, the lungs were inflated with $4 \%$ PFA by gravity inflation with a pressure of $20 \mathrm{~cm} \mathrm{H}_{2} \mathrm{O}$.

MLC-S2O fluorescence analysis. Paraffin-embedded sections $(8-\mu \mathrm{m})$ from the apex, middle, and base of lungs from 4 control and $4 \mathrm{Pbx} 1 / 2$-CKO mice were stained by immunofluorescence for phosphorylated MLC-S20, imaged using a $\times 20$ objective, and analyzed with ImageJ. The VSM ring area was determined by measuring the difference between the area of the outer circumference (solid line) and the inner circumference (dashed line) of the MLC-S20-stained VSM cells (Supplemental Figure 6). The fluorescence intensity of each MLC-S20-positive VSM ring was determined by measuring the difference in fluorescence intensity between the outer and inner rings, divided by the area of that ring. The average MLC-S20 fluorescence intensity was calculated for each mouse and then averaged within each group. Data are presented as the average fluorescence intensity for each group, normalized to the control values \pm SEM, with statistical comparisons made using a Student's $t$ test. Because fluorescence intensity varies significantly between experiments, each comparison experiment was performed simultaneously, with all antibody incubations, washes, and imaging conducted in a single batch by a single investigator. The results presented for each comparison represent a single, complete experiment. Each experiment was repeated a minimum of 3 times so as to confirm each result.

Computation of PVR. The predicted PVR response to treatment with the Rho-kinase inhibitor Y-27632 relative to PBS-treated control mice $\left(\mathrm{PVR} / \mathrm{PVR}_{\mathrm{CTL}}\right.$ ) was calculated using histological vessel size data from control mice treated with Y-27632, Pbx1/2-CKO mice treated with PBS, and Pbx1/2-CKO mice treated with Y-27632. The PVR was assumed to be partitioned in the arterial, venous, and microcirculatory compartments in control conditions according to prior literature (58). Assuming that Y-27632 acts to dilate small arteries and that loss of PBX does not alter the number of small arteries, the PVR relative to the control in each condition can be calculated according to Poi- 
seuille's law: PVR/PVR ${ }_{\text {CTL }}=\left[2+\left(D_{\text {Avg CTL }} / D_{\text {Avg }}\right)^{4}\right] / 3$, where $D_{\text {Avg }}$ is the mean diameter of small arteries in each sample (small arteries were those with 10 or fewer nuclei). The average predicted PVR was calculated for each mouse and then averaged within the groups. Data are presented as the average predicted PVR normalized to control values \pm SEM, with statistical analysis done using a Student's $t$ test for comparisons between 2 groups or 2-way ANOVA with Tukey's method for comparisons between 2 groups under 2 or more conditions.

In vivo pulmonary artery pressure measurement. Four control mice were anesthetized with urethane $(1 \mathrm{~g} / \mathrm{kg})$, intubated, and placed on a ventilator (Harvard Apparatus) on a heated pad. A bilateral thoracotomy was performed, and a 1.0-Fr high-fidelity pressure catheter (Millar Inc.) was inserted into the right ventricle. The pressure tracing was recorded and analyzed on commercially available software (Notocord). After establishing the baseline recordings, $30 \mu \mathrm{l}$ PBS was injected into the superior vena cava. The subsequent changes were recorded 30 seconds after this injection. Mice were then administered an identical volume of Y-27632 (50 mg/ $\mathrm{kg}$ ) by injection into the superior vena cava. The subsequent changes were recorded 30 seconds after this injection, and data were averaged over a 30-second period for each condition. The average RVSP was calculated for each mouse at baseline, after treatment with PBS, and after treatment with Y-27632. The data are reported as the average RVSP \pm SEM, with statistical comparisons made using 1-way ANOVA with Tukey's method to adjust for multiple comparisons.

Statistics. Statistical comparisons were made for each experiment using a minimum of 3 samples for each group. Comparisons between 2 groups were made using a 2-tailed Student's $t$ test. Comparisons among 3 groups were made using 1-way ANOVA with Tukey's method to adjust for multiple comparisons. Comparisons between 2 groups under 2 or more conditions were made using a 2-way ANOVA with Tukey's method to adjust for multiple comparisons. All data are represented as the mean \pm SEM. A $P$ value of 0.05 or less was considered statistically significant.

Study approval. Mice were housed and all experimental procedures were performed in an American Association for Accreditation of Laboratory Animal Care-accredited laboratory animal facility at the University of Wisconsin - Madison. This study was approved by the
University of Wisconsin - Madison Institutional Animal Care and Use Committee and adhered to the NIH's Guide for the Care and Use of Laboratory Animals (National Academies Press, 2011).

\section{Author contributions}

DJM devised and performed the experiments, composed the figures, and wrote the manuscript. MDW helped devise and perform the experiments and contributed to the preparation of the figures. EAH conducted the initial Pbx1/2-CKO genetic cross. TAH and AR performed the echocardiographic measurements. RJP and NCC contributed to the mathematical modeling of pulmonary pressure based on vessel size. RZ and LS provided the $P b x 1$-floxed and $P b x 2$-null mice and contributed to the experimental design and writing of the manuscript. XS is the senior author and contributed to the experimental design and completion of the manuscript.

\section{Acknowledgments}

We would like to thank Wei Shi (Keck School of Medicine, USC) and Mark Lewandoski (Center for Cancer Research, National Cancer Institute) for sharing mouse strains; the Laboratory for Optical and Computational Instrumentation (LOCI) at the University of Wisconsin - Madison for assistance with image reconstruction; Jens Eickhoff for review of the statistical analyses; Ellen Wald, Akihiro Ikeda, Marlowe Eldridge, De-Ann Pillers, Jim Gern, Jill Wildonger (all from the University of Wisconsin - Madison), and members of the Sun laboratory for thoughtful discussions and review of the manuscript. This work was supported by a Translational Science Career Development Award (KL2TR000428, to DJM); a subaward from the National Center for Advancing Translational Sciences (NCATS) grant to the University of Wisconsin - Madison Institute for Clinical and Translational Research (UL1TR000427); NIH grants (OT2OD023857, NHLBI RO1 HL113870, HL097134, and HL122406, to XS); and a grant from the Wisconsin Partnership Program (2897, to XS and DJM).

Address correspondence to: Xin Sun, Department of Pediatrics, UCSD, 9500 Gilman Dr., La Jolla, California 92093, USA. Phone: 858.246.2000; Email: xinsun@ucsd.edu.
1. Balayla J, Abenhaim HA. Incidence, predictors and outcomes of congenital diaphragmatic hernia: a population-based study of 32 million births in the United States. JMatern Fetal Neonatal Med. 2014;27(14):1438-1444.

2. McGivern MR, et al. Epidemiology of congenital diaphragmatic hernia in Europe: a register-based study. Arch Dis Child Fetal Neonatal Ed. 2015;100(2):F137-F144.

3. Lally KP, et al. Standardized reporting for congenital diaphragmatic hernia--an international consensus. J Pediatr Surg. 2013;48(12):2408-2415.

4. Wynn J, et al. Outcomes of congenital diaphragmatic hernia in the modern era of management. J Pediatr. 2013;163(1):114-9.e1.

5. Coughlin MA, et al. Prenatally diagnosed severe CDH: mortality and morbidity remain high. J Pediatr Surg. 2016;51(7):1091-1095.

6. Dillon PW, Cilley RE, Mauger D, Zachary C, Meier A. The relationship of pulmonary artery pressure and survival in congenital diaphragmatic hernia. J Pediatr Surg. 2004;39(3):307-312; discussion 307.

7. Lusk LA, Wai KC, Moon-Grady AJ, Steurer MA, Keller RL. Persistence of pulmonary hypertension by echocardiography predicts short-term outcomes in congenital diaphragmatic hernia. JPediatr. 2015;166(2):251-256.e1.

8. Bos AP, Tibboel D, Koot VC, Hazebroek FW, Molenaar JC. Persistent pulmonary hypertension in high-risk congenital diaphragmatic hernia patients: incidence and vasodilator therapy. J Pediatr Surg. 1993;28(11):1463-1465.

9. Kinsella JP, et al. Randomized, multicenter trial of inhaled nitric oxide and high-frequency oscillatory ventilation in severe, persistent pulmonary hypertension of the newborn. J Pediatr. 1997;131(1 Pt 1):55-62.

10. Putnam LR, et al. Evaluation of variability in inhaled nitric oxide use and pulmonary hyper- tension in patients with congenital diaphragmatic hernia. JAMA Pediatr. 2016;170(12):1188-1194.

11. Mercier JC, Lacaze T, Storme L, Rozé JC, Dinh-Xuan AT, Dehan M. Disease-related response to inhaled nitric oxide in newborns with severe hypoxaemic respiratory failure. French Paediatric Study Group of Inhaled NO. Eur J Pediatr. 1998;157(9):747-752.

12. Derderian SC, Jayme CM, Cheng LS, Keller RL, Moon-Grady AJ, MacKenzie TC. Mass effect alone may not explain pulmonary vascular pathology in severe congenital diaphragmatic hernia. Fetal Diagn Ther. 2016;39(2):117-124.

13. Akinkuotu AC, et al. Are all pulmonary hypoplasias the same? A comparison of pulmonary outcomes in neonates with congenital diaphragmatic hernia, omphalocele and congenital lung malformation. J Pediatr Surg. 2015;50(1):55-59.

14. Jay PY, et al. Impaired mesenchymal cell function in Gata4 mutant mice leads to diaphragmatic 
hernias and primary lung defects. Dev Biol. 2007;301(2):602-614.

15. Ackerman KG, Wang J, Luo L, Fujiwara Y, Orkin $\mathrm{SH}$, Beier DR. Gata4 is necessary for normal pulmonary lobar development. Am J Respir Cell Mol Biol. 2007;36(4):391-397.

16. Ackerman KG, et al. Fog2 is required for normal diaphragm and lung development in mice and humans. PLoS Genet. 2005;1(1):58-65.

17. You LR, et al. Mouse lacking COUP-TFII as an animal model of Bochdalek-type congenital diaphragmatic hernia. Proc Natl Acad Sci U S A. 2005;102(45):16351-16356.

18. Guilbert TW, Gebb SA, Shannon JM. Lung hypoplasia in the nitrofen model of congenital diaphragmatic hernia occurs early in development. Am J Physiol Lung Cell Mol Physiol. 2000;279(6):L1159-L1171.

19. Keijzer R, Liu J, Deimling J, Tibboel D, Post M. Dual-hit hypothesis explains pulmonary hypoplasia in the nitrofen model of congenital diaphragmatic hernia. Am J Pathol. 2000;156(4):1299-1306.

20. Yu L, et al. De novo copy number variants are associated with congenital diaphragmatic hernia. JMed Genet. 2012;49(10):650-659.

21. Yu L, et al. Variants in GATA4 are a rare cause of familial and sporadic congenital diaphragmatic hernia. Hum Genet. 2013;132(3):285-292.

22. Merrell AJ, Ellis BJ, Fox ZD, Lawson JA, Weiss JA, Kardon G. Muscle connective tissue controls development of the diaphragm and is a source of congenital diaphragmatic hernias. Nat Genet. 2015;47(5):496-504.

23. Longoni $\mathrm{M}$, et al. Prevalence and penetrance of ZFPM2 mutations and deletions causing congenital diaphragmatic hernia. Clin Genet. 2015;87(4):362-367.

24. Penkov D, et al. Analysis of the DNA-binding profile and function of TALE homeoproteins reveals their specialization and specific interactions with Hox genes/proteins. Cell Rep. 2013;3(4):1321-1333.

25. Capellini TD, Zappavigna V, Selleri L. Pbx homeodomain proteins: TALEnted regulators of limb patterning and outgrowth. Dev Dyn. 2011;240(5):1063-1086.

26. Russell MK, et al. Congenital diaphragmatic hernia candidate genes derived from embryonic transcriptomes. Proc Natl Acad Sci U S A. 2012;109(8):2978-2983.

27. Schnabel CA, Selleri L, Jacobs Y, Warnke R, Cleary ML. Expression of Pbx1b during mammalian organogenesis. Mech Dev. 2001;100(1):131-135.

28. Naiche LA, Arora R, Kania A, Lewandoski M, Papaioannou VE. Identity and fate of Tbx4expressing cells reveal developmental cell fate decisions in the allantois, limb, and external genitalia. Dev Dyn. 2011;240(10):2290-2300.

29. Selleri L, et al. The TALE homeodomain protein $\mathrm{Pbx} 2$ is not essential for development and long-term survival. Mol Cell Biol. 2004;24(12):5324-5331.

30. Li W, et al. Pbx1 activates Fgf10 in the mes- enchyme of developing lungs. Genesis. 2014;52(5):399-407.

31. Branchfield K, Li R, Lungova V, Verheyden JM, McCulley D, Sun X. A three-dimensional study of alveologenesis in mouse lung. Dev Biol. 2016;409(2):429-441.

32. Neptune ER, et al. Dysregulation of TGF-beta activation contributes to pathogenesis in Marfan syndrome. Nat Genet. 2003;33(3):407-411.

33. Shifren A, Durmowicz AG, Knutsen RH, Hirano E, Mecham RP. Elastin protein levels are a vital modifier affecting normal lung development and susceptibility to emphysema. Am J Physiol Lung Cell Mol Physiol. 2007;292(3):L778-L787.

34. Thibault HB, et al. Noninvasive assessment of murine pulmonary arterial pressure: validation and application to models of pulmonary hypertension. Circ Cardiovasc Imaging. 2010;3(2):157-163.

35. Urboniene D, Haber I, Fang YH, Thenappan T, Archer SL. Validation of high-resolution echocardiography and magnetic resonance imaging vs. high-fidelity catheterization in experimental pulmonary hypertension. Am J Physiol Lung Cell Mol Physiol. 2010;299(3):L401-L412.

36. Kitagawa M, Hislop A, Boyden EA, Reid L. Lung hypoplasia in congenital diaphragmatic hernia. A quantitative study of airway, artery, and alveolar development. Br J Surg. 1971;58(5):342-346.

37. Geggel RL, Murphy JD, Langleben D, Crone RK, Vacanti JP, Reid LM. Congenital diaphragmatic hernia: arterial structural changes and persistent pulmonary hypertension after surgical repair. JPediatr. 1985;107(3):457-464.

38. Sheikh AQ, Misra A, Rosas IO, Adams RH, Greif DM. Smooth muscle cell progenitors are primed to muscularize in pulmonary hypertension. $\mathrm{Sci}$ Transl Med. 2015;7(308):308ra159.

39. Zhang W, et al. Spatial-temporal targeting of lung-specific mesenchyme by a Tbx 4 enhancer. BMC Biol. 2013;11:111.

40. Keller RL, Moore P, Teitel D, Hawgood S, McQuitty J, Fineman JR. Abnormal vascular tone in infants and children with lung hypoplasia: Findings from cardiac catheterization and the response to chronic therapy. Pediatr Crit Care Med. 2006;7(6):589-594.

41. Shimokawa $\mathrm{H}$, et al. Anti-anginal effect of fasudil, a Rho-kinase inhibitor, in patients with stable effort angina: a multicenter study. JCardiovasc Pharmacol. 2002;40(5):751-761.

42. Vicari RM, et al. Efficacy and safety of fasudil in patients with stable angina: a double-blind, placebo-controlled, phase 2 trial. J Am Coll Cardiol. 2005;46(10):1803-1811.

43. Skinner JR, Boys RJ, Hunter S, Hey EN. Non-invasive assessment of pulmonary arterial pressure in healthy neonates. Arch Dis Child. 1991;66(4 Spec No):386-390.

44. Abman SH, Chatfield BA, Hall SL, McMurtry IF. Role of endothelium-derived relaxing factor during transition of pulmonary circulation at birth. Am J Physiol. 1990;259(6 Pt 2):H1921-H1927.

45. Raj U, Shimoda L. Oxygen-dependent signaling in pulmonary vascular smooth muscle. Am J Physiol Lung Cell Mol Physiol. 2002;283(4):L671-L677.

46. Hanson KA, Burns F, Rybalkin SD, Miller JW, Beavo J, Clarke WR. Developmental changes in lung cGMP phosphodiesterase-5 activity, protein, and message. Am J Respir Crit Care Med. 1998;158(1):279-288.

47. Thibeault DW, Haney B. Lung volume, pulmonary vasculature, and factors affecting survival in congenital diaphragmatic hernia. Pediatrics. 1998;101(2):289-295.

48. Iocono JA, Cilley RE, Mauger DT, Krummel TM, Dillon PW. Postnatal pulmonary hypertension after repair of congenital diaphragmatic hernia: predicting risk and outcome. JPediatr Surg. 1999;34(2):349-353.

49. Keller RL, et al. Congenital diaphragmatic hernia: endothelin-1, pulmonary hypertension, and disease severity. Am J Respir Crit Care Med. 2010;182(4):555-561.

50. Bos AP, Tibboel D, Hazebroek FW, Stijnen T, Molenaar JC. Congenital diaphragmatic hernia: impact of prostanoids in the perioperative period. Arch Dis Child. 1990;65(9):994-995.

51. Patel N, Moenkemeyer F, Germano S, Cheung MM. Plasma vascular endothelial growth factor A and placental growth factor: novel biomarkers of pulmonary hypertension in congenital diaphragmatic hernia. Am J Physiol Lung Cell Mol Physiol. 2015;308(4):L378-L383.

52. Longoni M, et al. Molecular pathogenesis of congenital diaphragmatic hernia revealed by exome sequencing, developmental data, and bioinformatics. Proc Natl Acad Sci U S A. 2014;111(34):12450-12455.

53. Yu L, et al. Whole exome sequencing identifies de novo mutations in GATA6 associated with congenital diaphragmatic hernia. J Med Genet. 2014;51(3):197-202.

54 . Yu L, et al. Increased burden of de novo predicted deleterious variants in complex congenital diaphragmatic hernia. Hum Mol Genet. 2015;24(16):4764-4773.

55. Koss $\mathrm{M}$, et al. Congenital asplenia in mice and humans with mutations in a Pbx/Nkx2-5/p15 module. Dev Cell. 2012;22(5):913-926.

56. Branchfield K, Nantie L, Verheyden JM, Sui P, Wienhold MD, Sun X. Pulmonary neuroendocrine cells function as airway sensors to control lung immune response. Science. 2016;351(6274):707-710.

57. Harris SP, et al. Hypertrophic cardiomyopathy in cardiac myosin binding protein- $\mathrm{C}$ knockout mice. Circ Res. 2002;90(5):594-601.

58. Hakim TS, Michel RP, Chang HK. Partitioning of pulmonary vascular resistance in dogs by arterial and venous occlusion. J Appl Physiol Respir Environ Exerc Physiol. 1982;52(3):710-715. 